\title{
Lungenszintigraphie (Version 3)
}

\section{Überarbeitet: 2.4.2017, 31.8.2017, 09.11.2017 und 06.04.2018}

\author{
Carl Schümichen ${ }^{1}$; Matthias Schmidt ${ }^{2}$; Thomas Krause ${ }^{3}$ \\ ${ }^{1}$ vormals Klinik und Poliklinik für Nuklearmedizin, Universität Rostock; \\ ${ }^{2}$ Klinik für Nuklearmedizin, Universitätsklinikum Köln; \\ ${ }^{3}$ Universitätsklinik für Nuklearmedizin, Inselspital, Universität Bern
}

\section{Schlüsselwörter}

Leitlinie, Lungenszintigraphie, VIP-SPECT, V/P-SPECT/CT, akute Lungenembolie, CTEPH, Voraussage der postoperativen Lungenfunktion

\section{Zusammenfassung}

In der aktualisierten und erweiterten S1-Leitlinie zur Lungenszintigraphie werden die Vorzüge der Methode beim Nachweis der akuten Lungenembolie (LE) in der Lungenperipherie (subsegmentale LE), bei subakuten und chronischen Grunderkrankungen der Lunge, sowie beim Nachweis der chronischen LE (CTEPH) herausgestellt. Methode der Wahl ist die Ventilations/Perfusions(V/P)-SPECT oder V/P-SPECT/CT mit nochmals gesteigerter Spezifität. Aufgrund der hohen Sensitivität wird, um eine Übertherapie zu vermeiden, ein Schwellenwert eingeführt (V/P-mismatch in mindestens einem Segment oder zwei Subsegmenten). Im Falle eines veränderten Therapieverhaltens (nur Observation anstelle Antikoagulation) kann der Schwellenwert entfallen. Zur klinisch-therapeutischen Wertigkeit von subsementalen LE werden neue Daten präsentiert, die Kapitel offene Fragen wurden erweitert. Die weiteren Indikationen für V/P-SPECT (Nebendiagnosen, Anomalien der Lungenperfusion, Voraussage der postoperativen Lungenfunktion) werden mit neueren Daten hinterlegt.

\section{Keywords}

German guidelines, lung scintigraphy, VIP SPECT, V/P SPECT/CT, acute pulmonary embolism, CTEPH, prediction of postoperative lung function

\section{Summary}

The S1 guideline for lung scintigraphy has been updated and extended in order to emphasize the advantages oft the method in detecting acute pulmonary embolism (PE) in the periphery oft the lung (subsegmental PE), in underlying subacute and chronic pulmonary disorders, as well as in detecting chronic LE (CTEPH). Method of choice is ventilation / perfusion (V/P) SPECT or V/P SPECT/CT with even higher specificity. Because of its high sensitivity, a threshold (V/P mismatch in at least one segment or two subsegments) is introduced to avoid overtreatment. In case of a change in the therapeutic approach (observation only instead of anticoaculation) the threshold can be omitted. New data concerning the clinical and therapeutical impact of subsegmental PE are included, the chapters open questions have been extented. Other indications for VIP SPECT (secondary diagnoses, abnormalities in pulmonary perfusion, prediction of postoperative lung function) are presented with new data.

Lung scintigraphy

Nuklearmedizin 2018; 57: 74 - 90

Korrespondenzadresse

Deutsche Gesellschaft für Nuklearmedizin

Nikolaistraße 29

37073 Göttingen

Tel.: +49 (0)551 48857-401

Fax: +49(0)551 48857-401

E-Mail: office@nuklearmedizin.de

\section{Zielsetzung}

Die nuklearmedizinische Leitlinie zur Lungenszintigraphie wurde aktualisiert und erweitert, vorrangig wird unter Einbeziehung neuer Daten die akute Lungenembolie (LE) behandelt. Eine ausführliche Risikobewertung der akuten LE erklärt den sich anbahnenden Paradigmenwechsel in der Behandlung normotensiver Patienten (ausschließlich ambulante Therapie oder nur Beobachtung anstelle Antikoagulation). Methodisch werden V/P-SPECT und V/P-SPECT/CT als heutiger Standard angesehen, Alternativen (nur P-SPECT oder P-SPECT/CT) sind nur im Notfall angemessen. Die Befundungskriterien von V/P-SPECT wurden (unter Berücksichtigung der EANM-Leitlinie) überarbeitet. Die diagnostische Wertigkeit von subsegmentalen LE wird, soweit möglich, mit Daten belegt, der hieraus abgeleitete Schwellenwert wird diskutiert, auch unter dem Gesichtspunkt eines veränderten Therapieverhaltens. Neu aufgenommen wurde das Kapitel chronische LE bzw. pulmonalarterielle Hypertonie/CTEPH. Die aktuellen Referenzwerte und die Daten zur Strahlenexposition wurden überprüft und aktualisiert, veraltete Methoden (radioaktive Edelgase) entsorgt. Angeborene und erworbene Anomalien der Lungen und Lungenstrombahn incl. Shuntbestimmungen und insbesondere die Voraussage der postoperativen Lungenfunktion werden unter Berücksichtigung neuer Daten abgehandelt. Das Literaturverzeichnis wurde insgesamt erweitert, um den Leser in die Lage zu versetzen, die Methode auch argumentativ zu vertreten. 


\section{Hintergrundinformatio- nen zur Lungenembolie}

Die akute Lungenembolie (LE) entsteht durch eine partielle oder vollständige Verlegung der Lungenarterien durch eingeschwemmte Thromben aus der peripheren venösen Blutstrombahn. Einzelne oder wiederholte Ereignisse können zur chronischen LE führen.

In Deutschland versterben jährlich etwa 40000 Patienten an einer akuten LE, unter den häufigsten Todesursache steht die LE derzeit nach der Sepsis an vierter Stelle. Bis zu $90 \%$ aller Todesfälle sollen sich innerhalb der ersten 2 Stunden nach Symptombeginn ereignen (112). Ein nicht genau bezifferbarer Anteil der Patienten verstirbt ohne Diagnostik und adäquate Therapie. Bei unklarer Todesursache liegt autoptisch bei $40-80 \%$ eine LE zugrunde $(33,111$, 136, 137). Die Frühletalität ist abhängig vom Ausmaß der LE (Thrombuslast), und mehr noch vom Vorliegen und Schweregrad einer rechtsventrikulären Dysfunktion (27). Kardiopulmonale und maligne Begleiterkrankungen erhöhen sowohl kurzals auch langfristig das Mortalitätsrisiko um mehr als das Doppelte $(41,90)$.

Die Mortalität während des stationären Aufenthaltes steigt bis auf $22 \%$, wenn intensivmedizinische Maßnahmen (120) oder eine Thrombolyse (101) erforderlich werden. Im Durchschnitt aller Schweregrade beträgt die Mortalitätsrate knapp 4\% der Patienten $(26,90,156)$. Während der ambulanten Weiterbehandlung erhöht sich das Mortalitätsrisiko durch thromboembolische Folgeereignisse und Nebenwirkungen der Therapie, sowie durch Rechtsherzversagen bei Patienten mit persistierender pulmonaler Hypertonie. Die kumulative Mortalität nach 30 Tagen, bezogen auf alle Schweregrade, wird im Mittel mit etwas mehr als $10 \%$ angegeben $(4,12,26,58,90)$.

Nach überstandener symptomatischer LE besteht ein lange anhaltendes Morbiditätsrisiko. Die Lebensqualität der Betroffenen ist deutlich beeinträchtigt (141). Innerhalb von 12 Monaten werden bei knapp einem Drittel der Patienten erneute Perfusionsdefekte (Rezidive) beobachtet, das körperliche Befinden (Luftnot, kürzere Laufstrecke) ist beeinträchtigt, der Pulmonalisdruck erhöht (119). Das Risiko bezüglich
Mortalität, symptomatischer LE-Rezidive, kardiovaskulärer Ereignisse sowie Entwicklung einer chronisch-thrombembolischen pulmonalen Hypertonie (CTEPH) ist nach vier Jahren gegenüber einer Kontrollgruppe um das Dreifache erhöht (65). Die Folgerisiken können wahrscheinlich durch eine frühzeitige Diagnose und damit auch einer früheren Therapie der LE minimiert werden (119). Andererseits können die Folgerisiken mit Hinblick auf Mortalität, residuale Dyspnoe und RV-Dysfunktion durch eine thrombolytische Therapie, die zusätzlich zu einer Heparinbehandlung gegeben wird, nicht reduziert werden (67).

Patienten, die bei der stationären Aufnahme primär kreislaufstabil (normotensiv) sind, weisen per se eine deutlich bessere Prognose auf. In den USA betrifft dies ca. 75\% Patienten (122). Nur bei diesen Patienten wird regelhaft vor Therapie eine bildgebende Diagnostik zum Nachweis der LE durchgeführt. Nach szintigraphischer Diagnose versterben während des stationären Aufenthaltes lediglich $1 \%$ und nach CTPA-Diagnose nur 2\% der Patienten (54), nach 30 Tagen sind es 6\% (27). Aufgrund der relativ günstigen Prognose eröffnen sich neue Möglichkeiten im Umgang mit der Erkrankung. So kann die Behandlung aus den stationären in den ambulanten Bereich verlagert werden, nahezu die Hälfte aller Patienten mit akuter LE wäre hierfür geeignet (78). Unter ambulanter oraler Antikoagulation reduziert sich die Komplikationsrate (Rezidiv, größere Blutung oder Tod) in den ersten 14 Tagen und den folgenden 3 Monaten bei allen klinischen Schweregraden um mehr als den Faktor drei (115). Eine weitere, in den USA zunehmend genutzte Option ist die stationäre Aufnahme des Patienten lediglich zur Beobachtung, eine Antikoagulation erfolgt nur fakultativ (146). Die stationäre Aufenthaltsdauer reduziert sich um die Hälfte, die Rezidivrate in den folgenden zwei Monaten unterscheidet sich nicht gegenüber der konventionellen Behandlung (146).

Aus dem Mortalitätsrisiko wird die Notwendigkeit einer unverzüglichen und konsequent durchgeführten Diagnostik bei klinischem Verdacht auf eine akute LE abgeleitet.

\section{Definitionen}

A. Aerosol-Ventilationsszintigraphie: Maß für die regionale Ventilation $(V)$ ist die bronchoalveoläre Deposition von inhaliertem Radio-Aerosol innerhalb der Lungen.

B. Gas-Ventilationsszintigraphie:

$\mathrm{Maß}$ für die regionale Ventilation (V) ist die bronchoalveoläre Verteilung eines radioaktiven Gases während der Atmung.

C. Lungen-Perfusionsszintigraphie:

$\mathrm{Maß}$ für die regionale Perfusion $(\mathrm{P})$ ist die Verteilung intravenös applizierter Makropartikel in der Lungenendstrombahn.

D. V/P-planar:

Zusammenschau sowohl der regionalen Perfusion als auch Ventilation der Lungen in planarer Aufnahmetechnik.

E. V/P-SPECT:

Zusammenschau sowohl der regionalen Perfusion als auch Ventilation der Lungen in tomographischer Aufnahmetechnik.

F. V/P-SPECT/CT:

Bildgebendes Hybridverfahren zur Beurteilung der regionalen Perfusion, Ventilation sowie der Morphologie der Lungen in tomographischer Aufnahmetechnik.

G. CTPA:

Computertomographische Pulmonalisangiographie

\section{Indikationen}

1. Diagnostik der akuten Thromboembolie der Lunge (LE) vorzugsweise bei kreislaufstabilen Patienten, gleichrangig zur CTPA. In der Notfallmedizin sind SPECT/CT und ein 24-Std.-Service anzustreben. Vorrangige Indikation in folgenden Situationen:

- Erhöhtes Kreatinin (i.e. Niereninsuffizienz), Kontrastmittelunverträglichkeit, Hyperthyreose, ersetzt die CTPA

- LE-Symptomatik und negativer CTPA-Befund

- Junge Frauen, geringere Strahlenexposition der Mammae als bei der CTPA 

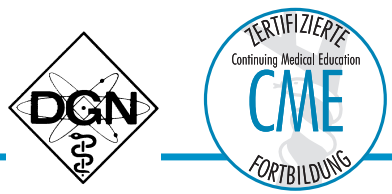

Tab. 1 Scores zur Bestimmung der klinische Wahrscheinlichkeit einer Lungenembolie $(64,71,147)$. TVT $=$ tiefe Beinvenenthrombose; $\mathrm{LE}=$ Lungenemboli

\begin{tabular}{|c|c|c|c|}
\hline Variable & $\begin{array}{l}\text { Revidierter } \\
\text { Genfer Score }\end{array}$ & $\begin{array}{l}\text { Vereinfachter } \\
\text { Genfer Score }\end{array}$ & Wells Score \\
\hline Frühere TVT oder LE & 3 & 1 & 1,5 \\
\hline Haemoptysen & 2 & 1 & 1,5 \\
\hline Aktive Krebserkrankung & 2 & 1 & 1 \\
\hline $\begin{array}{l}\text { Herzfrequenz } \\
75-94 / \mathrm{min} \\
\geq 95 / \mathrm{min} \\
>100 / \mathrm{min}\end{array}$ & $\begin{array}{l}1 \\
2\end{array}$ & $\begin{array}{l}1 \\
2\end{array}$ & 1,5 \\
\hline $\begin{array}{l}\text { Operation oder Knochenfraktur innerhalb des } \\
\text { letzten Monats }\end{array}$ & 2 & 1 & \\
\hline $\begin{array}{l}\text { Operation innerhalb } 1 \text { Monat oder Immobilisati- } \\
\text { on } \geq 3 \text { Tage }\end{array}$ & & & 1,5 \\
\hline Einseitiger Beinschmerz & 3 & 1 & \\
\hline $\begin{array}{l}\text { Schmerzen bei tiefer Palpation der unteren } \\
\text { Extremität und einseitiges Beinödem }\end{array}$ & 4 & 1 & \\
\hline $\begin{array}{l}\text { Objektivierbare klinische Zeichen und Symptome } \\
\text { einer TVT }\end{array}$ & & & 3 \\
\hline $\begin{array}{l}\text { Wahrscheinlichkeit für eine LE > als für } \\
\text { Differenzialdiagnosen }\end{array}$ & & & 3 \\
\hline \multicolumn{4}{|l|}{ Klinische Wahrscheinlichkeit (3-Stufen-Score) } \\
\hline Niedrig & $0-3$ & $0-1$ & $0-1,5$ \\
\hline Mittel & $4-10$ & $2-4$ & $2-6$ \\
\hline Hoch & $\geq 11$ & $\geq 5$ & $>6$ \\
\hline
\end{tabular}

- Schwangerschaft und auch post partum, geringere Strahlenexposition der proliferierenden Mammae

- Geschlechtsunabhängig bei jüngeren Patienten, geringeres Karzinomrisiko in Folge

2. Bei allen Patienten mit pulmonaler Hypertonie unklarer Ursache zum Ausschluss oder Nachweis einer chronisch thromboembolischen pulmonalen $\mathrm{Hy}$ pertonie (CTEPH)

3. Zur seitengetrennten Quantifizierung der Ventilation und Perfusion präoperativ vor Lungenresektion bei Bronchialkarzinomen, chirurgischer oder endoskopischer Lungenvolumenreduktion bei COPD, Lungentransplantation oder Lungenresektion bei nichtmalignen Erkrankungen (z.B. lokalisierte Bronchiektasen)

4. Diagnostik und Differenzierung angeborener und erworbener Anomalien der Lungen incl. relativer Perfusionsverteilung bei Stenosen der Pulmonalgefäße,
Quantifizierung eines Rechts-LinksShunts.

5. Die Beurteilung und Quantifizierung pulmonaler Selbstreinigungsmechanismen, wie die resorptive und mukoziliäre Clearance, wurden nicht in diese Leitlinie aufgenommen, da sie keinen Eingang in die klinische Routine gefunden haben.

\section{Untersuchungsmethode, Vorgehensweise, Verfahren}

\section{A. Vorabinformationen bei der Fragestellung Lungenembolie}

\section{Auswahl der Patienten}

Bei Verdacht auf Lungenembolie soll eine initiale Risikostratifizierung erfolgen, um zwischen hämodynamisch stabilen und instabilen Patienten zu unterscheiden, dies ist Aufgabe des Klinikers. In aller Regel werden nur stabile Patienten einer szintigraphischen Abklärung zugeführt. Sollte der

Patient unmittelbar vor oder während der Diagnostik instabil werden oder bei instabilen Patienten von vornherein eine CTPA nicht in Frage kommen, so sind besondere Verhaltensmaßregeln $\mathrm{zu}$ beachten (siehe Kapitel E.3.4.).

\section{Klinische Wahrscheinlichkeit}

Bei stabilen Patienten sollte zunächst eine Einschätzung der klinischen Wahrscheinlichkeit (KW) für das Vorliegen einer Lungenembolie erfolgen. Hierzu stehen verschiedene Scores zur Verfügung: der Wells Score (147) und in Europa auch der revidierte Genfer Score (71). Die Scores beurteilen die Vortestwahrscheinlichkeit nach einem Punktsystem ( Tabelle 1). Präziser aber auch wesentlich aufwändiger ist ein Voraussagemodell, das 16 Variable erfasst, die sowohl positiv als auch negativ mit einer LE assoziiert sind (84). Alternativ kann eine untersucherbasierte empirische Beurteilung herangezogen werden. Häufig unterschätzt wird die subjektiv wahrgenommene klinische Wahrscheinlichkeit durch folgende Umstände: Hohes Patientenalter, bekannte Herzinsuffizienz, chronische Lungenerkrankung, Antikoagulation sowie eine fehlende Verfahrensanweisung (116).

\section{Klinischer Befund}

Die klinischen Symptome einer akuten LE sind unspezifisch, häufig sind Dyspnoe mit plötzlichem Beginn, Tachykardie, Thoraxschmerz und Synkope oder Präsynkope, gegebenenfalls auch Hämoptyse. Der physikalische Befund an der Lunge ist in der Regel unauffällig.

\section{Basisdiagnostik}

Zur Basisdiagnostik zählen die Vitalparameter, eine Röntgen-Thoraxübersicht in 2 Ebenen, ein EKG und die Blutgasanalyse. Jeder Parameter für sich betrachtet ist nicht aussagekräftig, ausschlaggebend ist der Gesamteindruck. Die Anfertigung einer Röntgen-Thoraxübersichtsaufnahme sollte eine indizierte V/P-SPECT-Untersuchung nicht verzögern. 


\section{D-Dimer-Test}

Bei hämodynamisch stabilen Patienten soll ein D-Dimer-Test nur nach vorheriger Einschätzung der klinischen Wahrscheinlichkeit durchgeführt werden. Bei niedriger und mittlerer KW und normalen D-Dimeren ist keine weitere LE-Diagnostik erforderlich. Erhöhte D-Dimere sind kein Beweis für eine LE, diese muss durch ein bildgebendes Verfahren bestätigt oder ausgeschlossen werden. Bei hoher KW soll ein D-Dimer-Test nicht eingesetzt werden, da sein negativ-prädiktiver Wert in dieser Situation nicht ausreichend hoch ist (98, 125). Besonders niedrig ist die Spezifität bei älteren ( $>80$ Jahre) oder hospitalisierten Patienten, bei Patienten mit malignen Tumoren und in der Schwangerschaft. Ein altersbezogener D-Dimer Cut-off bei Patienten über 50 Jahre (Alter in Jahren x $10 \mu \mathrm{g} / \mathrm{ml}$ ) verringert den Anteil der Patienten, der einer bildgebenden Diagnostik zugeführt werden muss (114).

\section{Tiefe Beinvenenthrombose}

Bei einem Patienten mit hoher klinischer Wahrscheinlichkeit bzw. bei nicht-hoher klinischer Wahrscheinlichkeit und positivem $\mathrm{D}$-Dimer-Test kann der positive Nachweis einer tiefen Beinvenenthrombose (TVT) ohne weitere bildgebende Diagnostik als gleichbedeutend mit einer Bestätigung des Verdachts auf LE angesehen werden. Die Indikation zur Antikoagulation ist ohnehin gegeben, gleichzeitige, asymptomatische Lungenembolien sind in einem hohen Prozentsatz nachweisbar. Szintigraphisch haben zwei Drittel aller Patienten mit TVT Perfusionsausfälle in der Lunge, $(89,92,100)$, in der CTPA werden bei einem Drittel (14) bzw. bei zwei Drittel (28) der Patienten gleichzeitige Lungenembolien gefunden. Die Behandlung auch asymptomatischer Emboli soll Überlebensvorteile bringen (19).

\section{CTPA}

Wird eine Bildgebung erforderlich, wird gegenwärtig mehrheitlich eine CT-Pulmonalisangiographie (CTPA) durchgeführt. Die CTPA ist universell verfügbar und liefert rasch einen Befund. Mit Weiterent- wicklung der Spiral-CT zur Multidetektor(zeilen)-CT (MDCT) wurde der negativ prädiktive Wert ständig verbessert, nach negativem Befund und ohne Behandlung über drei Monate ging die Häufigkeit von symptomatischen Folgeembolien von anfänglich $1,4 \%$ (fatal $0,51 \%$ ) (87) ab der 64-Zeilen Generation auf $0 \%$ zurück (105). Nichtokkludierende Emboli, auch im Truncus pulmonalis, werden sicher erkannt. Die CTPA ist vor allem geeignet, zentral gelegene Embolien zu erkennen, wohingegen die CTPA Schwächen aufweist, periphere Emboli nachzuweisen, anstelle des erwünschten Direktnachweises des Embolus („Füllungsdefekt") tritt der weniger spezifische „Gefäßabbruch“. Die Interobserver-Variabilität beim Nachweis von subsegmentalen LE (SSLE) ist hoch $(16,83)$.

\section{V/P-Szintigraphie}

Die Ventilations-/Perfusions-(V/P)Szintigraphie kann bei hämodynamisch stabilen Patienten als vollwertige Alternative zur CTPA eingesetzt werden. Sie wird besonders empfohlen, wenn die CTPA bei individueller Abschätzung zu risikoreich erscheint: schwere Niereninsuffizienz, schwere Iod-Kontrastmittelallergie, junge Frauen oder in der Schwangerschaft und auch post partal $(2,9,74,104)$. Sie ist auch geeignet zum sicheren Ausschluss einer Lungenembolie bei geringer Symptomatik. Als indirektes Nachweisverfahren („Perfusionsdefekt mit lobärer, segmentaler oder subsegmentaler Gestalt") wird eine ausreichend hohe Spezifität nur durch die $\mathrm{Zu}$ sammenschau mit der regionalen Ventilation erzielt („Perfusionsdefekt bei erhaltener Ventilation"). Die Sensitivität und Spezifität von V/P-SPECT wird in einer Metaanalyse von 3454 Patienten mit $96 \%$ und $97 \%$ angegeben (59). Die Spezifität ist mit der Hybridbildgebung V/P-SPECT/CT nochmals steigerungsfähig (36). Prinzipbedingt kann die Szintigraphie auch Emboli auf der Subsegmentebene erfassen (123). Diagnostisch limitierend beim Nachweis der LE sind nicht okkludierende oder teilokkludierende Emboli, die vor allem in den zentralen Abschnitten des Pulmonalarterienbaums auftreten. Hier gilt es, nicht nur Perfusionsdefekte sondern auch relative Perfusionsminderungen $\mathrm{zu}$ erkennen. Bereits mit der planaren V/P-Szintigraphie wird ein hoher negativer prädiktiver Wert erreicht, die Rate symptomatischer Rezidive bei negativem Befund und dreimonatiger Nachbeobachtung der unbehandelten Patienten beträgt $0 \%$ (32).

\section{Gegenüberstellung V/P-SPECT/ CTPA}

In einer Metaanalyse von 5923 Patienten wird die durchschnittliche diagnostische Treffsicherheit von V/P-SPECT und CTPA als gleichwertig beurteilt (106). In einer weiteren Metaanalyse wird V/P-SPECT/CT als überlegene Methode herausgestellt: Sensitivität $97,6 \%$ vs. $82,0 \%$, Spezifität $96,9 \%$ vs. $94,9 \%$ (42). In einer Metaanalyse der Cochrane Collaboration werden die Ergebnisse der Szintigraphie (verschiedene Techniken) und der CTPA während der Schwangerschaft gegenübergestellt: Sensitivität $100 \%$ vs. $83 \%$, inkonklusive Ergebnisse $4,0 \%$ vs. $5,9 \%$ (143). Die Vorzüge einer höheren Sensitivität zeigen sich in folgenden Situationen:

- Im Direktvergleich diagnostiziert V/P-SPECT in der Summe 18\% mehr Embolien als die CTPA in der mittleren Befundungskategorie (48).

- Bei Lungenfibrose als Grunderkrankung und Nachweis einer LE ist V/P-SPECT der CTPA deutlich überlegen (Sensitivität $100 \%$ versus 22\%) (75).

- In der Diagnostik einer chronisch thromboembolischen pulmonalen Hypertonie (CTEPH) wird der szintigraphischen Diagnostik ein hoher Stellenwert eingeräumt (150) (Kapitel I.).

Vorteile der szintigraphischen Diagnostik werden auch bei der Strahlenexposition deutlich, die in einer Metanalyse 2,12 mSv für V/P-SPECT und 4,94 $\mathrm{mSv}$ für die CTPA betragen soll (106). Anders als bei der Szintigraphie ist die Bandbreite der Strahlenexposition durch eine Thorax-CT sehr hoch. Die mitgeteilten Dosen reichen von $4,8 \mathrm{mSv}$ (153) über 6,4-6,8 mSv, mit einer Variationsbreite bis 2,1 (45) bis hin $\mathrm{zu}$ mehr als doppelt so hohen Werten: $13,2 \mathrm{mSv}$ (24), $11,8 \mathrm{mSv}$ (79) und $11 \mathrm{mSv}$ (131). Von den beinflussbaren Variablen hat das Untersuchungsprotokoll eine grö- 
ßere Bedeutung als der Gerätetyp (131). Der Überschreitungsgrad der anatomischen Lungengrenzen ist eine weitere Variable, es können die Schilddrüse mit 5,1 $\mathrm{mSv}$ und die Mammae mit 1,5 mSv zusätzlich belastet werden (153). Sehr hohe Dosen, bis im Mittel $22 \mathrm{mSv}$ in der älteren Literatur (46), wurden mit der Warnung vor einer Karzinominduktion in den besonders exponierten Mammae verbunden (23, 46). Die Mammadosis wird mit 20-50 mSv beziffert (9). Eine Abschirmung der Mammae reduziert die Dosis im Mittel um 42,1\% (113). Effektiver ist eine Modulation des Röhrenstromes, die mittlere Dosis wird von $51,5 \mathrm{mSv}$ auf $8,6 \mathrm{mSv}$ gesenkt (117). Vergleichsweise geringe $0,6 \mathrm{mSv}$ werden für P-SPECT und $0,8 \mathrm{mSv}$ für V/P-SPECT angegeben (9). Unter diesem Aspekt wird bei jungen Frauen und in der Schwangerschaft vorrangig die szintigraphische Diagnostik empfohlen (2, 9, 74, 104), ebenso in der post partalen Periode (22). Bei Schwangeren sind die potenziellen Risiken für die Mutter (Kapitel A.10.) und das Kind (nachfolgend) gegeneinander abzuwägen. Wird gemäß der Europäischen Leitlinie (8) ausschließlich eine P SPECT mit reduzierter Aktivität $(50 \mathrm{MBq})$ durchgeführt, beträgt die fetale Dosis 0,11-0,20mGy $(18,93)$. Die fetale Dosis nach CT, anfänglich mehr als doppelt so hoch (47), soll aktuell unter 0,1 mGy bleiben (151). Die genannten Dosen sind kleiner als regionale Unterschiede in der natürlichen Strahlenexposition innerhalb von Deutschland.

Aufgrund der genannten Vorzüge hat sich in Institutionen mit geeigneter apparativer Ausstattung die Szintigraphie als First-Line-Untersuchung erhalten oder erneut etabliert.

\section{Indikationsstellung bei jüngeren Patienten}

Die von einer Thorax-CT ausgehende Strahlenexposition ist aktuell gegenüber V/P-SPECT um den Faktor 2-5 erhöht. Ein erhöhtes Karzinom-Risiko infolge einer diagnostischen Strahlenexposition wurde bei Kindern (102) und auch bei Jugendlichen (80) numerisch belegt. Nach CT-Untersuchungen verschiedener Regionen mit einer durchschnittlichen Dosis von 4,5mSv fand sich im Kollektiv von 680000 Patienten verschiedener Altersgruppen eine im Mittel um den Faktor 1,24 erhöhte Inzidenz von Karzinomen (80). Die Inzidenz nahm mit der Anzahl der Untersuchungen zu und war umso höher, je jünger der Patient zum Untersuchungszeitpunkt war. Für das Mamma-Ca war mit einem Faktor von 0,99 ein solcher Zusammenhang nicht nachweisbar. Auch beim hoch belasteten fliegenden Personal konnte bei hohen Fallzahlen (bis 93771) eine signifikant erhöhte Mortalität durch ein Mamma-Ca nicht belegt werden $(37,154)$. Die noch bestehenden Unsicherheiten bezüglich der Risikobewertung von CT betreffen die Dosimetrie und insbesondere die Auswahl der Patienten und müssen durch internationale Studien ausgeräumt werden (11). Bis zur Klärung des Sachverhaltes wird weiterhin eine Schonung der Mammae empfohlen, zumal aufgrund des sonstigen möglichen Folgerisikos die Empfehlung zur Szintigraphie geschlechtsunabhängig auf alle jüngeren Patienten ausgedehnt werden kann.

\section{Subsegmentale LE}

Wird bei Autopsiebefunden präzise zwischen zentraler (proximal bis einschließlich Segmentarterie) und peripherer (distal der Segmentarterien) Lokalisation der Emboli sowie zwischen LE als Todesursache $(29,2 \%)$ und LE als Nebendiagnose $(70,8 \%)$ unterschieden, so führen bei knapp einem Viertel (22,6\%) der Betroffenen ausschließlich subsegmental lokalisierte Emboli zum Tode (111).

Von sämtlichen Emboli sind 74\% ausschließlich in der Peripherie lokalisiert (111), die im Vergleich zur CTPA relative Häufung szintigraphischer Befunde auf der Subsegmentebene spiegelt die tatsächlichen Verhältnisse wider.

Zur Risikobewertung isolierter subsegmentaler LE ist die Studienlage noch unzureichend (152), unklar ist auch, wie viele falsch positive Befunde der CTPA zu einer Verharmlosung beitragen (83). Andererseits weisen sowohl mit der CTPA $(19,108$, 140) als auch szintigraphisch (30) diagnostizierte SSLE im Verlauf eine erhöhte Rate tiefer Beinvenenthrombosen auf. Zumindest bei Karzinompatienten ist auch das Mortalitätsrisiko erhöht, es wird eine Anti- koagulation empfohlen. Eine vierfach erhöhte Mortalität ist gesichert bei Patienten mit hohem Wells Score (1). Ansonsten wäre eine weitere Option die sofortige Abklärung einer tiefen Beinvenenthrombose und nur im positiven Fall die Einleitung einer Therapie (51). Hier besteht noch Klärungsbedarf.

\section{2. Überdiagnostik und Übertherapie}

Seit Einführung der CTPA im Jahr 1989 hat in den USA die Inzidenz der LE und die Rate damit assoziierter Nebenwirkungen der Therapie stetig zugenommen, die Mortalität pro diagnostiziertem Fall hat abgenommen, nicht jedoch die krankheitsbezogene Mortalität in der Gesamtbevölkerung (148). Verantwortlich hierfür wird eine zunehmend verbesserte Sensitivität der CTPA und in diesem Zusammenhang auch der Wechsel von V/P-planar (sensitivitätsmindernd in den USA nach PIOPED-Kriterien ausgewertet) auf die unter diesen Umständen relativ höher auflösende CTPA gemacht (128). Hierdurch sollen zunehmend mildere Fälle mit LE diagnostiziert werden, die von einer Therapie weniger profitieren.

In den USA konnte zwischen 1999 bis 2008 die fallbezogene Mortalität zwar reduziert werden, die LE-assoziierte Gesamtmortalität hat dagegen leicht zugenommen. Ursache hierfür ist eine exzessive Ausweitung der Diagnostik (124). Hierbei werden nicht zunehmend mildere Fälle sondern alle Schweregrade erfasst (88). Patienten, die bisher ohne Diagnose und adäquate Therapie verstarben $(33,111,136$, 137) und die bisher, von den wenigen autoptisch gesicherten Fällen abgesehen, nicht in die Mortalitätsstatistik als LE-bezogen eingegangen sind, werden vermehrt der LE als Todesursache zugeordnet. Ein möglicher positiver Einfluss der exzessiven Diagnostik auf die Gesamtmortalität wird nicht wahrgenommen (124). In vielen anderen Industrieländern mit mehr zurückhaltender Vorgehensweise wurde eine Abnahme der LE-assoziierten Gesamtmortalität in den vergangenen zehn oder mehr Jahren nachgewiesen: Australien (129), Kanada und mehre europäische Länder (E, F, S, DK) $(70,95,132)$. Für Deutschland lie- 
gen vor 2004 widersprüchliche Daten vor (44), zwischen 2004 und 2011 ist die Mortalität um 24,3\% zurückgegangen (107).

Das Thema Übertherapie ist noch nicht abgeschlossen. Bei kreislaufstabilen (normotensiven) Patienten bringt die stationäre Beobachtung (ohne Therapieabsicht) mit verkürzter Aufenthaltsdauer offensichtlich identische Behandlungsergebnisse wie die stationär durchgeführte konventionelle Therapie (146). Langzeitergebnisse liegen noch nicht vor.

\section{B. Informationen für die Durchfüh- rung der Untersuchung}

1. Für eine risikoarme Durchführung und sowie für eine korrekte Befundung der V/P-Szintigraphie sollten aus der klinischen Vorgeschichte folgende Informationen erhoben werden: (chronisch) obstruktive Atemwegserkrankung, Rechtslinks-Shunt, schwere pulmonale Hypertonie, Linksherzversagen und intravenöser Drogen-Abusus.

2. Die aktuellen Untersuchungsergebnisse des Thorax, evtl. einschließlich Röntgenbild, der Beinvenen und, falls durchgeführt, der D-Dimer-Bestimmung sollten bereits vor Beginn der Szintigraphie vorliegen. Die klinische Untersuchung in der Nuklearmedizin konzentriert sich auf vitale Symptome, insbesondere auf die Atmung und den Blutdruck. Falls eine aktuelle Röntgenthorax-Untersuchung nicht vorliegt, sollte eine indizierte V/P-SPECT bzw. V/P-SPECT/CT hierdurch nicht verzögert werden.

3. Das Röntgen-Thoraxbild ist bei Patienten mit Lungenembolie in der Mehrzahl der Patienten diagnostisch nicht zielführend. Zur Differenzialdiagnostik ist auf der aktuellen p.-a. oder a.-p. und ggf. auch seitlichen Röntgen- Thoraxaufnahme auf Verdichtungen, Atelektasen, Ergüsse, Raumforderungen, eine Kardiomegalie und regional verminderte pulmonale Gefäßzeichnung zu achten.

4. Überprüfung, ob Vorbefunde einer LEDiagnostik vorliegen.

5. Während einer Schwangerschaft wird als Eingangstest die (V)/P-Szintigraphie empfohlen $(2,9,74,104)$.

\section{Vorsichtsmaßregeln}

Ein Markierungskit enthält je nach Hersteller 1,5-2 Millionen MAA-Partikel. Die Anzahl der applizierten MAA-Partikeln kann sich kritisch auf die Hämodynamik auswirken, ein Maximalwert von 700000 Partikeln sollte nicht überschritten werden. Bei Patienten mit pulmonaler Hypertonie oder Rechts-Links-Shunt sowie bei Jugendlichen und Kindern sollte die Partikelzahl limitiert werden (siehe Kapitel V.D.2.2.). Insgesamt sollen nicht mehr als 0,1\% der Lungenkapillaren embolisiert werden. Bei Erwachsenen kann die Anzahl der Partikel bis auf 150000 Partikel reduziert werden, ohne dass die Diagnostik durch eine inhomogene Aktivitätsverteilung zu sehr beeinträchtigt wird. Zur Klärung eines Shunts wird die Partikelzahl auf 20000 begrenzt (Risiko von Krampanfällen).

\section{Radiopharmaka und Aktivitäts- dosierung}

Folgende Substanzen stehen zur Verfügung

\section{Ventilation}

\subsection{Mittelfeines Aerosol (flüssige oder solide Partikel)}

Tc-99m-DTPA in Form flüssiger Partikel wird wegen der raschen pulmonalen Clearance (T1/2 55 Min) (86) und der anschließend renalen Ausscheidung bei Kindern bevorzugt. Die intrapulmonale Verweildauer von soliden Tc-99m Nanokolloiden wird vom Depositionsort beeinflusst, der wiederum von der Partikelgröße und von der Flussrate während der Inhalation beeinflusst wird. Die Partikel werden innerhalb der ersten 24 Stunden ausschließlich aus den Zilien tragenden großen und kleinen Luftwegen (0-12 Generation des Bronchialbaums) geklärt. Der Anteil ungünstiger Partikel mit einem Durchmesser $>2 \mu \mathrm{m}$ (vorzeitige Deposition in Stenosen infolge von Turbulenzen) ist abhängig von der Qualität der Partikelselektion im Radioaerosolgenerator und daher gerätetypisch. Es sind zahlreiche Geräte im Handel. Üblicherweise werden $1000 \mathrm{MBq}$ (diagnostischer Referenzwert) bis $1100 \mathrm{MBq}$ (Höchstwert) Tc-99m-Pertechnetat in den Radioaerosol-Generator gegeben, wovon etwa 20 bis $50 \mathrm{MBq}$ in den Lungen deponiert werden. Referenzwerte für pädiatrische Untersuchungen liegen nicht vor. Es wird jedoch auf die jeweils aktuellen Empfehlungen der EANM verwiesen (21).

\subsection{Ultrafeines Trockenaerosol}

Die mit Tc-99m markierten ultrafeinen Graphitpartikel (Technegas ${ }^{\circledast}$ ) werden überwiegend im Alveolarraum deponiert und weisen eine mittlere Residenzzeit von 5,6 Tagen in der Lunge auf (60). Der mittlere aerodynamische Durchmesser beträgt unmittelbar nach Generierung $<0,01 \mu \mathrm{m}$, ein ungünstiges Partikelwachstum durch $\mathrm{Zu}$ sammenprall und nachfolgender Verschmelzung der Partikel (Koagulation) tritt bei verlängerter Wartezeit bis zur Inhalation auf (Limit 10 Min.). Mehrfaches Eindampfen, obwohl vom Hersteller empfohlen, sollte wegen Zunahme hygroskopischer Eigenschaften mit sekundärem Partikelwachstum vermieden werden. Für den Fall einer zu geringen Aktivitätskonzentration im Eluat stehen größere Graphit-Tiegel zur Verfügung.

Es werden bis $350 \mathrm{MBq}$ (diagnostischer Referenzwert) - $500 \mathrm{MBq}$ (Höchstwert) Tc99m-Pertechnetat in den Radio-AerosolGenerator gegeben, wovon etwa 20 bis $50 \mathrm{MBq}$ in den Lungen deponiert werden. Referenzwerte für pädiatrische Untersuchungen liegen nicht vor. Es wird auch hier auf die jeweils aktuellen Empfehlungen der EANM verwiesen (21).

Die Aerosol-Ventilationsszintigraphie wird vor der Perfusionsszintigraphie durchgeführt, da es aufgrund des niedrigen Wirkungsgrades der Radioaerosolgeneratoren praktisch unmöglich ist, eine Aktivität von Tc-99m Aerosol in der Lunge zu deponieren, die drei- bis viermal größer ist als die von Tc-99m MAA. Sollte man, wie zum Beispiel in der Schwangerschaft empfohlen, auf eine Ventilationsaufnahme verzichten und diese nach der PerfusionsSzintigraphie erforderlich werden, kann diese am Folgetag nachgeholt werden.

\subsection{Xenon-133}

Xe-133 wird im deutschsprachigen Raum nicht mehr verwendet. 


\subsection{Krypton- $81 \mathrm{~m}$}

$\mathrm{Kr}-81 \mathrm{~m}$ wird aus einem $\mathrm{Rb}-81 / \mathrm{Kr}$ $81 \mathrm{~m}-\mathrm{Generator}$ gewonnen (HWZ von Rb-81 4,58Std). Auch Kr-81m wird im deutschsprachigen Raum nicht mehr verwendet.

\section{Perfusion}

\subsection{Tc-99m MAA}

Mit Tc-99m markiertes makroaggregiertes Albumin (MAA) ist das Radiopharmakon der Wahl. Die biologische Halbwertzeit von Tc-99m MAA in der Lunge liegt zwischen 1,5 bis 3 Stunden. Bei Erwachsenen beträgt der diagnostische Referenzwert bei planarer Aufnahmetechnik $100 \mathrm{MBq}$, der Höchstwert $125 \mathrm{MBq}$; bei SPECT ist der Referenzwert $160 \mathrm{MBq}$, der Höchstwert $200 \mathrm{MBq}$, bei Kindern 0,5-2 MBq/kg mit einem Minimum von $10 \mathrm{MBq}$. Dosimetrie siehe $>$ Tabelle 2 (Erwachsene) und $>$ Tabelle 3 (Kinder). In der Schwangerschaft ist unter Berücksichtigung der fetalen Strahlenexposition die Untersuchung mit reduzierter Aktivität $(50 \mathrm{MBq})$ durchzuführen. Idealerweise sollte das Aktivitätsverhältnis Perfusion/Ventilation $\geq 4$ betragen. Die Anzahl der Partikel sollte sich zwischen 200000-700000 bewegen. Bei Kindern ist die Anzahl der Partikel dem Gewicht anzupassen:

- <10 kg 10000-50000,

- 10-20kg 50000-150000,

- 20-35kg 150000-300000,

- $35-50 \mathrm{~kg} 500000$.

Markierte MAA-Partikel setzen sich im Reaktionsgefäß ab, das deshalb vor der Aktivitätsentnahme geschüttelt werden soll. Für Kinder gilt die jeweils aktuelle EANMDosiskarte.

\section{E. Datenakquisition}

\section{Untersuchungsablauf}

Regelhaft wird die kombinierte V/P-Szintigraphie mit der Ventilation begonnen und danach die Perfusion durchgeführt, beide Untersuchungen in SPECT-Technik. Eine Ausnahme in der Abfolge ergibt sich nur bei Anwendung von Kr-81m aufgrund unterschiedlicher keV-Energien. Ist hierbei die zuerst durchgeführte Perfusionsszintigraphie normal oder entspricht dem Rönt-

Tab. 2 Dosimetrie bei Erwachsenen

\begin{tabular}{l|l|l|l}
\hline & $\begin{array}{l}\text { Applizierte Aktivität } \\
(\mathrm{MBq})\end{array}$ & $\begin{array}{l}\text { Kritisches Organ } \\
(\mathrm{mGy} / \mathrm{MBq})\end{array}$ & $\begin{array}{l}\text { Effektive Dosis } \\
(\mathrm{mSv} / \mathrm{MBq})\end{array}$ \\
\hline Tc-99m-MAA & & 0,066 Lunge & 0,011 \\
\hline Tc-99m-DTPA $^{2}$ & $40-200$ & 0,062 Blase & 0,0049 \\
\hline${\text { Tc-99m } \text { Technegas }^{3}}^{20-50}$ & $20-50$ & 0,11 Lunge & 0,015 \\
\hline Kr-81 $^{4}$ & $40-400$ & 0,00021 Lunge & 0,000027 \\
\hline
\end{tabular}

${ }^{1}$ ICRP 80, S. 69 (50); ${ }^{2}$ ICRP 80, S. 59 (50); ${ }^{3}$ ICRP 80, S. 33 (50); ${ }^{4}$ ICRP 53, S. 160 (49)

Tab. 3 Dosimetrie bei Kindern (5 Jahre)

\begin{tabular}{|c|c|c|c|}
\hline Radiopharmakon & $\begin{array}{l}\text { Applizierte Aktivität } \\
\text { (MBq/kg) }\end{array}$ & $\begin{array}{l}\text { Kritisches Organ } \\
\text { (mGy/MBq) }\end{array}$ & $\begin{array}{l}\text { Effektive Dosis } \\
\text { (mSv/MBq) }\end{array}$ \\
\hline Tc-99m-MAA ${ }^{1}$ & $0,5-2$ & 0,20 Lunge & 0,034 \\
\hline Tc-99m-DTPA² & $0,4-0,6$ & 0,095 Blase & 0,0090 \\
\hline Tc-99m Technegas 3 & $10-12$ & 0,33 Lunge & 0,047 \\
\hline $\mathrm{Kr}-81 \mathrm{~m}^{4}$ & $0,5-5$ & 0,00068 Lunge & 0,000088 \\
\hline
\end{tabular}

${ }^{1}$ ICRP 80, S. 69 (50); ${ }^{2}$ ICRP 80, S. 59 (50); ${ }^{3}$ ICRP 80, S. 33 (50); ${ }^{4}$ ICRP 53, S. 160 (49)

gen-Thoraxbild, kann die Ventilationsszintigraphie entfallen.

Wird die Tc-99m-Aerosol-Szintigraphie vor der Perfusionsszintigraphie durchgeführt, so muss die in den Lungen deponierte Aktivität von Radioaerosol auf höchstens ein Viertel der Aktivität von Tc-99m MAA begrenzt werden. Ein Aktivitätsverhältnis Ventilation zu Perfusion von 1:3 ist als noch tolerable Untergrenze anzusehen. In der Praxis wird dieser Mindestwert bei einem Viertel der Patienten nicht erreicht (121). Wurde versehentlich eine $\mathrm{zu}$ hohe Aktivität von Radioaerosol in der Lunge deponiert, so begründet dies eine entsprechende Erhöhung der Aktivität von Tc-99m MAA über den diagnostischen Höchstwert hinaus. Während der Schwangerschaft kann, um die Strahlenexposition zu minimieren, die Untersuchung mit $\mathrm{P}$ SPECT begonnen werden, gegebenenfalls bei positivem Befund am nächsten Tag ergänzt durch V SPECT (7-9).

\subsection{Aerosol-Ventilationsszintigraphie}

Vorzugsweise inhaliert der Patient das Aerosol in aufrechter Position, hierdurch wird bei Dyspnoe und erst recht bei Orthopnoe die Inhalation erleichtert, die Lungen sind besser belüftet. Für eine bessere Vergleich- barkeit mit der Perfusionsuntersuchung kann die Inhalation auch im Liegen erfolgen $(7,8)$. Das Aerosol wird über ein Mundstück bei verschlossener Nase oder über eine Maske appliziert. Kinder und selbst komatöse Patienten können mit Technegas über einen Ambu-Beutel mit leichtem Überdruck beatmet werden. Der Patient soll entweder durch eigene Kontrolle oder durch eine gesteuerte Flussregulation (z. B. Stenose) ganz langsam tief einatmen, um turbulente Strömungen an Abzweigungen im Bronchialsystem oder durch Stenosen zu minimieren. Nach dem Einatmen sollte die Luft kurz angehalten werden, um die Deposition der Aerosolpartikel im Bronchialsystem und insbesondere im Alveolarraum zu erhöhen. Ein Monitoring der inhalierten Aktivität mit einem Handdosimeter wird empfohlen, um diese nach oben zu begrenzen. Die Gammakamera, mit der später die Aufnahmen gemacht werden, sollte hierfür wegen Kontaminationsgefahr nicht herangezogen werden. Die Aerolsol-Inhalation sollte zur Vermeidung einer Raumkontamination in einem gesonderten Raum und ggf. unter einem Abzug erfolgen. Nach Abschluss der Inhalation ultrafeiner Partikel (Technegas) wird noch bis zu zehn Minuten Radioakti- 
Tab. 4 Rekonstruktionsparameter (Beispiele)

\begin{tabular}{l|l|l|l}
\hline Algorithmus & Iterationen & Subsets & Filter \\
\hline OSEM & 4 & 8 & Butterworth; cutoff, 0.8 cycles/cm; order 9 \\
\hline Astonish & 4 & 8 & kein \\
\hline Flash 3D & 4 & 8 & Gauss $8 \mathrm{~mm} \mathrm{FWMH,} \mathrm{post} \mathrm{recon.,} \mathrm{Streuungskorrektur}$ \\
\hline
\end{tabular}

vität abgeatmet. Das Anlegen eines Mundschutzes ist nur bei mittelfeinem Aerosol wirksam, bei Technegas wäre ein HEPAFilter erforderlich.

Bei Patienten mit Atemwegsobstruktion kann ein turbulenter Fluss zur zentralen Aktivitätsdeposition und damit zu einem suboptimalen Ergebnis der Studie führen. Bei Anwendung von ultrafeinem Trockenaerosol wird dieser ungünstige Effekt minimiert. Das Ausmaß eines „reverse mismatch" soll kleiner und bei Patienten mit COPD sollen zusätzliche Mismatch Befunde besser erkannt werden (55).

Sollte ein Patient in Ausnahmesituationen nicht für die SPECT-Akquisition geeignet sein und die Untersuchung auf planare Aufnahmen beschränkt werden, so müssen Ventilations-Aufnahmen in sämtlichen Projektionen korrespondierend zu denen der späteren Perfusionsszintigraphie angefertigt werden. Mindestens 6 (RVL, LDR, RPO, LPO, Re Lat, Li Lat), besser 8 Sichten (+ RAO, + LAO) sollten akquiriert werden.

\subsection{Perfusionsszintigraphie}

Nachdem der Patient mehrfach tief durchgeatmet und der ältere Patient auch gehustet hat, wird Tc-99m-MAA langsam intravenös injiziert. Vor dem Injizieren sollte die Spritze geschüttelt werden. Das Rücksaugen von Blut ist zu vermeiden, damit es zu keiner Clotbildung kommt. Der Patient liegt auf dem Rücken oder sollte auch bei Orthopnoe so dicht wie möglich an diese Position herankommen. Die Szintigraphie wird üblicherweise in Rücken- und ggf. auch in Bauchlage durchgeführt, eine Mehrkopf-Gammakamera ist zu bevorzugen.

Nur bei schwierigem venösem Zugang kann ein zentralvenöser Zugang benutzt werden, der danach gut gespült werden soll, jedoch kein Swan-Ganz-Katheter (Kathederspitze liegt in der Arteria pulmona- lis, ab hier keine homogene Durchmischung der MAA-Partikel mehr gewährleistet) und kein venöser Zugang, der Filter (z.B. für eine Chemotherapie) enthält.

Es sei darauf hingewiesen, dass Zeugen Jehovas über die Injektion von Albumin als Bestandteil des Bluts aufzuklären sind.

\subsection{V/P-planar}

Die planare V/P-Szintigraphie sollte nur ausnahmsweise eingesetzt werden und bedarf der individuellen Begründung. Sie wird in multiplen Ansichten durchgeführt, welche die anteriore (RVL), posteriore (LDR), beide schräg-posterioren (RPO und LPO), beide schräg-anterioren (RAO und LAO) und beide lateralen Ansichten (Re Lat und Li Lat) einbeziehen. Optimum sind 8 Ansichten, Mindestzahl 6, sofern dies vom Patienten toleriert wird. Die schräg-anterioren oder die lateralen Projektionen können bei Bedarf entfallen. Im Einzel(not)fall ist jede Beschränkung in der Anzahl von Ansichten möglich.

Bei Patienten mit Kreislaufinstabilität (Schweregrad III nach ESC) und Kontraindikation zur CTPA wird geraten, sowohl auf eine Umpositionierung des Patienten als auch auf die Ventilations-Szintigraphie zu verzichten und lediglich ein Perfusionsszintigramm von anterior im Bett anzufertigen, gegebenenfalls ergänzt durch Aufnahmen von schräglateral.

\subsection{V/P-SPECT}

SPECT erhöht die Sensitivität der Methode $(10,110)$, reduziert die Interobserver-Varianz (10) und sollte deshalb heutiger Untersuchungsstandard sein. Das Potenzial von SPECT entfaltet sich nur dann, wenn Ventilation und Perfusion jeweils und dann mit identischer tomographischer Technik abgebildet werden. Unter Ausschöpfung der zulässigen diagnostischen Referenzwerte kann eine SPECT-Untersuchung mit einer Doppelkopfkamera in diagnostischer
Qualität innerhalb von ca. 25 Minuten durchgeführt werden.

\subsection{V/P-SPECT/CT}

Regelhaft wird die CT-Untersuchung direkt im Anschluss an die PerfusionsSPECT in unveränderter Position in Lowdose Technik durchgeführt. Einige Geräte sind nur auf diesen Modus ausgerichtet. Ein Low-dose CT ist primär für die anatomische Lokalisation gedacht, darüber hinausgehende Informationen müssen als Nebendiagnosen deklariert werden.

\section{Akquisitionsparameter}

\subsection{P planar, V/P-planar}

Matrix 256x256, 500-1000 kcounts pro Ansicht, High Resolution Low Energy Kollimator

\subsection{P-SPECT, V/P-SPECT}

Empfohlene Einstellung (Zwei-Kopf-Gamma-Kamera): High Resolution Low Energy Kollimator, Matrix 128x 128, 128 Projektionen (64 Projektionen pro Kopf), V: 12 Sek., P: 4Sek. pro Projektion.

Andere Parameter können je nach Kamera oder Patient sinnvoll sein:

General Purpose Kollimator, Matrix $128 \times 128,128$ Projektionen alternativ bei kurzen Aufnahmezeiten 64x64, (32 Projektionen pro Kopf), V: 10-20Sek., P: 4-10Sek. pro Projektion.

Beispiele für die Rekonstruktionsparameter finden sich in $>$ Tabelle 4.

\subsection{V/P-SPECT/CT}

Eine Low-dose-CT mit einer Strahlenexposition von maximal $1 \mathrm{mSv}$ ist meist ausreichend, z.B. mit einer Einstellung von $50 \mathrm{mAs}$ und $80 \mathrm{kV}$. Mit den neuen Rekonstruktionsverfahren (z.B. iterativ) kann die Strahlenexposition weiter optimiert werden, bzw. beim Cone-Beam-Verfahren der Bildkontrast verbessert werden. Auch mit älteren Geräten sollten die Parameter so gewählt werden, dass $2 \mathrm{mSv}$ nicht überschritten werden. Soll eine Bildfusion mit der SPECT durchgeführt werden, ist die (MD)CT bei angehaltenem Atem in Atemmittellage, eine Cone-Beam-Aufnahme während fortgesetzt leichter Atmung durchzuführen. Die (MD)CT in tiefer Inspiration führt zu einer besseren Entfal- 
tung der basalen Lungenabschnitte, andererseits zu einer fehlerhaften Bildfusion. Eine Kontrastmittelgabe ist nicht üblich.

\section{Interventionen}

Bei Patienten mit obstruktiver Atemwegserkrankung kann die vorherige Gabe eines Bronchodilatators die Aussagekraft der Untersuchung erhöhen.

\section{G. Datenauswertung}

Der Vergleich von regionaler Ventilation und Perfusion erfolgt visuell, kann aber auch anhand der dreidimensionalen SPECT-Daten mit eigens dafür entwickelten Programmen (semi)automatisiert werden, ein V/P-mismatch stellt sich dann im Positivkontrast dar $(10,109)$. Das Verfahren ist hilfreich für den weniger Erfahrenen, sowohl die Sensitivität als auch die Spezifität werden deutlich verbessert: Sensitivität Unerfahrene(r) visuell 0,75 , automatisiert 0,86, Erfahrene(r) visuell 0,94, automatisiert 0,96. Die entsprechenden Spezifitäten sind 0,86, 0,95 sowie 0,95, 0,97 (68).

Zur Voraussage der postoperativen Lungenfunktion wird bei planarer Aufnahmetechnik aus der ventralen und dorsalen Sicht das harmonische Mittel der gemessenen Impulse und hieraus das prozentuale Verhältnis rechte/linke Lunge bzw. von Teilen davon gebildet. Bei Anwendung von SPECT/CT ist eine Schwächungskorrektur vorzunehmen.

\section{H. Befundung und Dokumentation bei der Fragestellung akute LE}

\section{Grundlagen}

Aus der Zusammenschau von Ventilation und Perfusion ergeben sich verschiedene, diagnoserelevante Befundmuster:

V/P-mismatch ist der regionale, auf die anatomischen Grenzen eines Lappens, Segmentes oder Subsegmentes bezogene Perfusionsausfall bei regional erhaltener Ventilation. Er ist das szintigraphische Korrelat der Lungenembolie. Wichtigste und häufigste Differenzialdiagnose hierzu ist der

V/P-match mit regional gleichsinnig herabgesetzter Ventilation und Perfusion, in der Regel innerhalb der anatomischen Grenzen eines Lappens oder Segmentes (nicht Subsegmentes). Primär ist die Ventilation gestört, z.B. infolge einer Atemwegsobstruktion, der intakte v. Euler-Liljestrand Reflex verhindert sekundär einen pulmonalen Shunt. Seltener tritt ein

V/P-reverse-mismatch mit regional herabgesetzter Ventilation mit regional mäßig bis voll erhaltener Perfusion auf, entweder bei COPD mit gestörtem v. Euler-Liljestrand Reflex oder bei Pneumonie durch eine Mitreaktion der Vasa publica in Form einer entzündungsbedingten Hyperämie. Im letzteren Fall werden die anatomischen Grenzen nur bei der mittlerweile seltenen Lobärpneumonie eingehalten. Der V/P-reverse-mismatch ist immer gleichbedeutend mit einem funktionellen Shunt $\left(\mathrm{O}_{2}\right.$-Sättigung im Blut erniedrigt). Noch seltener aber zunehmend häufiger beobachtet wird ein

Pseudo-V/P-mismatch mit regional scheinbar mäßig bis deutlich gesteigerter Ventilation und regional normaler oder mäßig bis deutlich herabgesetzter Perfusion. Ursache ist eine entzündlich bedingte Einengung der Bronchiallumina (Peribronchitis), die verkürzte Diffusionstrecke sowie Turbulenzen führen zu einer verstärkten regionalen Radioaerosol-Deposition (auch und gerade bei Technegas). Die anatomischen Grenzen werden nicht eingehalten. Tatsächlich ist entgegen dem visuellen Eindruck die regionale Ventilation eingeschränkt. Der Befund ist zwischen V/P-match und V/P-reverse-mismatch einzuordnen, er findet sich gehäuft in den Unterfeldern.

Der triple-match-Befund setzt sich aus einem regionalen $\mathrm{V} / \mathrm{P}$-match-Befund mit einer Verschattung im Rö-Thoraxbild oder im CT an gleicher Stelle zusammen und soll für den Lungeninfarkt beweisend sein.

\section{P-planar, P-SPECT, P-SPECT/CT}

Steht die Ventilationsszintigraphie nicht zur Verfügung, zB. bei fehlender Mitarbeitsfähigkeit des Patienten Allgemeinzustand des Patienten, so muss sich die Diag- nose auf zusätzliche morphologische Merkmale des Perfusionsdefekts stützen. Gemäß PISA-PED Kriterien muss der Perfusionsdefekt exakt den anatomischen Lappen- bzw. Segmentgrenzen folgen (85). Im Vergleich von P-planar PISA-PED mit V/P-planar/CTPA als Referenz, V/P-planar sensitivitätsmindernd gemäß PIOPED IIKriterien bewertet fanden sich gleichwertige Ergebnisse (40), in einer anderen Studie wurde gegenüber V/P-SPECT mit P-planar nur bei $80 \%$ der Patienten eine diagnostische Aussage erzielt (130). Beim Vergleich von P-SPECT-PISA-PED mit V/P-SPECT als Referenz, letztere gemäß der Europäischen Leitlinie ausgewertet, soll eine Genauigkeit $\geq 90 \%$ erzielt werden (6). P-SPECT-PISA-PED ist jedoch nicht auf der Subsegmentebene einsetzbar, woraus sich insgesamt eine Einschränkung der Sensitivität ergibt, auf der Segmentebene ist zudem die Spezifität beeinträchtigt (124). Die alleinige Perfusionsszintigraphie wird nur noch für den Notfall empfohlen (5) und hat nur bei unauffälligem Befund einen sicheren diagnostischen Wert.

Die Kombination P-planar mit dem RöThoraxbild kann nicht empfohlen werden (PPV 71\%, NPV 83\%) (142). Mit der Hybridbildgebung P-SPECT/low-dose-CT wird, obwohl methodisch überlegenen, eine Spezifität von nur 51\% (36) bzw. $83 \%$ $(81,97)$ erzielt. Die CT kann, ähnlich wie das Rö-Thoraxbild, die Ventilationsszintigraphie nicht ersetzen.

\section{V/P-planar}

Die konventionelle planare V/P-Szintigraphie wurde durch die PIOPED I (Prospective Investigation of Pulmonary Embolism Diagnosis)-Studie (53) diskreditiert. Im direkten Vergleich mit der Pulmonalisangiographie wurde eine sehr hohe Sensitivität $(98 \%)$ bei einer extrem niedrigen Spezifität (10\%) ermittelt. Anstelle einer konkreten Aussage wurden verschiedene Befundkonstellationen katalogisiert und in fünf Wahrscheinlichkeitskategorien (PIOPED-Kategorien) mit unterschiedlich positiven Voraussagewerten eingeordnet.

Die PIOPED-Daten wurden mit Untersuchungstechniken gewonnen, deren Einzelfehler - bis zu $50 \%$ falsch positive Befunde mit der Single-breath-Xe-133-Venti- 
lationsszintigraphie, unzureichende $70 \%$ Sensitivität mit der Blattfilm-Pulmonalisangiographie, die zum damaligen Goldstandard erhoben wurde - sich zu einem massiven Gesamtfehler summiert haben (123). Eine Befundung nach PIOPED-Kriterien ist daher heutzutage obsolet.

Auch in Übereinstimmung mit der bisherigen Leitlinie (Version 2) und der Europäischen Leitlinie $(7,8)$ ist eine Befundung nach PIOPED-Kriterien aus europäischer Sicht abzulehnen. Auch im Ursprungsland der PIOPED-Befundung USA wird der Versuch unternommen, hiervon abzukommen (31).

Uneingeschränkte klinische Bedeutung hat nach wie vor der Normalbefund: Keine Perfusionsdefekte, das Perfusionsszintigramm entspricht von der Form dem Röntgenthoraxbild, das Ventilationsszintigramm kann abnormal sein (reverse mismatch). Dem Normalbefund wird ein sehr hoher negativ prädiktiver Wert zugeschrieben, die Folgeembolierate während einer dreimonatigen Nachbeobachtung beträgt 0\% (32). Dieses Ergebnis ist durch zahlreiche andere Kollektive gesichert. Evidenzgrad A, Level Ia $(7,8)$.

\section{V/P-SPECT}

Ein V/P-mismatch in den Segmenten 5 und 7 ist auch im gut einsehbaren Außenmantel der Lungen auf den vorgeschriebenen 6-8 planaren Ansichten nur wenig präsent. Ein V/P-mismatch auf Subsegmentebene wird auf planaren Ansichten praktisch nur im Außenmantel sichtbar. Der Zugewinn an Sensitivität durch Nachweis zusätzlicher V/P-mismatch Befunde mit SPECT beträgt auf der Segmentebene 12,8\%, auf der Subsegmentebene 82,6\% (110), im Mittel auf allen Ebenen 53,0\% (10). Entscheidend auch im Vergleich mit anderen bildgebenden Verfahren ist jedoch der Prozentsatz von $21 \%$, mit dem durch SPECT die Diagnose LE häufiger gestellt werden kann als mit der planaren Aufnahmetechnik (110). V/P-SPECT wird deshalb und auch wegen der geringeren Interobserver-Varianz gegenüber V/P-planar bevorzugt, Evidenzgrad B, Level IIb $(7,8)$ und soll heute als Untersuchungsmethode der Wahl angesehen werden.

\section{V/P-SPECT/CT}

Die Hybridbildgebung SPECT/CT wird in Analogie zum PET/CT die alleinige SPECT-Untersuchung zukünftig weitgehend ersetzen. Durch die CT-Komponente in low-dose Technik (V/P-SPECT/LDCT) erhöht sich bei der Fragestellung akute LE die Spezifität von $88 \%$ auf $100 \%$ bei einer konstant bleibenden Sensitivität von $97 \%$ (36). Auch ohne Kontrastmittel können mit LDCT wichtige Nebendiagnosen wie Pneumonie, Atelektasen, Infarkte, Tumore, Abszesse, Pleura- und Perikardergüsse und mit Einschränkung auch die Aortendissektion erkannt werden. Die niedrigere Sensitivität von Niedrigzeilen- und auch die von Cone-Beam-Geräten ist zu berücksichtigen. Mit LDCT finden sich alternative Diagnosen bei 27\% der Patienten (76), beim Einsatz der MDCT mit Kontrastmittel bis zu 40 \%. Trotz gewisser Einschränkungen kann V/P-SPECT/CT auch in der Notfalldiagnostik eingesetzt werden und dort die CTPA ersetzen.

Bei Patienten mit COPD bzw. bei gesunden Rauchern wird mit V/P-SPECT/ CT die Sicherheit der Interpretation in 9\% der Fälle durch die LDCT Komponente erhöht, bei $52 \%$ finden sich bei diesem Klientel zusätzliche Diagnosen, u.a. Tumor und Emphysem (56).

\section{Differenzialdiagnostik des V/P-mismatch}

Die V/P-Szintigraphie mit dem Kriterium „Perfusionsdefekt mit erhaltener Ventilation" ist als indirektes Nachweisverfahren der akuten LE anfällig für falsch positive Befunde. Die Ursachen für einen V/P-mismatch außer der akuten oder persistierenden LE sind vielfältig:

\section{Kompression der Pulmonalarterie}

Tumore aller Art, Lymphome und Narben, z.B. nach Tuberkulose. Beim Perfusionausfall eines ganzen Lungenflügels mit vollständigen oder auch teilweisen V/P-mismatch ist primär an ein zentrales Bronchial-Ca zu denken.

\section{Obstruktion der Pulmonalarterie}

Intraluminale Verstopfung durch verschiedene biologische und körperfremde Mate- rialien (3), u.a. auch durch Knochenzement nach Vertebroplastie (29) sowie durch Polyacrylamid Hydrogel nach kosmetischer intramuskulärer Anwendung (52). Beide Materialien sind im CT und Röntgenbild sichtbar.

\section{Vaskulitis}

Am häufigsten bei Takayasu, selten auch bei Sturge-Strauss Vaskulitis. Der V/P-mismatch kann erster Hinweis auf die Erkrankung sein (17).

\section{Obliteration der Pulmonalarterie}

Relativ häufig nach Strahlentherapie (99), auf die anatomischen Lappen- und Segmentgrenzen ist $\mathrm{zu}$ achten.

\section{Interpretation der Befunde}

Die klinische Frage nach einer Lungenembolie ist entweder positiv $\mathrm{zu}$ beantworten oder andernfalls zu verneinen, Wahrscheinlichkeitsdiagnosen sind abzulehnen. Sofern sich aus der Anamnese, den Vorbefunden und der begleitenden Röntgendiagnostik einschließlich CT kein Hinweis auf eine andere Ursache für einen V/P-mismatch ergibt, ist jeder typisch keilförmige Perfusionsdefekt mit erhaltener Ventilation als Lungenembolie zu werten. Atypisch geformte Perfusionsdefekte, die nicht den anatomische Lappen- und Segmentgrenzen folgen, sind zu ignorieren. Bei einem V/P-mismatch, der eine gesamte Lunge betrifft, sollte ein zentraler Lungentumor durch CT ausgeschlossen werden. Um eine Übersensitivität der szintigraphischen Diagnostik durch die empfohlene SPECTTechnik zu vermeiden, wird in Übereinstimmung mit der Europäischen Leitlinie $(7,8)$ ein mismatch-Befund von mehr als einem Subsegment gefordert, das heißt entweder ein Defekt von der Größe zweier Subsegmente oder zwei Defekte von Subsegmentgröße.

Bei planarer Aufnahmetechnik wird weiterhin ein einziger V/P-mismatch, der von der Größe und Form einem Subsegment entspricht, als beweisend für eine LE angesehen. Ein mit SPECT nachgewiesener singulärer V/P-mismatch auf der Subsegmentebene kann in Ausnahmefällen bei guter Übereinstimmung mit der Klinik und mit entsprechendem Vermerk im Be- 
fund ebenfalls als LE deklariert werden. In jedem Fall sollte ein solcher Befund dem Kliniker mitgeteilt und, sofern noch nicht geschehen, der sonographische Ausschluss einer tiefen Beinvenenthrombose veranlasst werden (siehe auch die Kapitel A.11. subsegmentale LE und VII. offene Fragen). Anmerkung: Das akzessorische Segment S3x weist grundsätzlich nur die Größe eines Subsegments auf, hier ist eine Fehldeutung im Sinne eines falsch negativen Befundes möglich.

Als LE positiv sind auch teilokkludierende Emboli zu betrachten, durch die sich Segmente oder ganze Lappen im Perfusions-Szintigramm noch schwach kontrastieren. Das Phänomen darf nicht verwechselt werden mit V/P-mismatch Befunden, bei denen durch Nichteinhaltung des optimalen Aktivitätsverhältnisses zu viel Aktivität von der Aerosol-Deposition in die Perfusion durchscheint. Teilokkludierende Emboli können mit SPECT besser erkannt und eingeordnet werden als mit planarer Aunahmetechnik. Im Zweifelsfall ist eine quantitative Analyse der SPECT-Daten hilfreich (38).

Der Befundbericht sollte Angaben darüber enthalten, wie viele Befunde mit V/P-mismatch vorhanden sind und auf welcher Ebene im pulmonalen Arterienbaum sie angesiedelt sind. Basiert die Diagnose Lungenembolie ausschließlich auf Befunden auf der Subsegmentebene, sollte dies aus dem Befundbericht eindeutig hervorgehen.

\section{LE positiv (V/P-SPECT)}

- V/P-mismatch in mindestens einem Segment oder zwei Subsegmenten, welcher der pulmonalen Gefäßanatomie entspricht. Die Anatomie der Lunge muss bekannt sein, weiterführende Literatur $(7,8)$.

\section{LE negativ}

- Normales Perfusionsverteilungsmuster, den anatomischen Grenzen der Lungen entsprechend

- V/P-match oder reverse match Befunde jeder Größe, Form oder Anzahl in Abwesenheit von mismatch Befunden

- V/P-mismatch Befunde, die keiner lobären, segmentalen oder subsegmentalen Form entsprechen.

\section{Nichtdiagnostisch}

- Multiple V/P-Abnormalitäten, die nicht mit einer spezifischen Erkrankung in Verbindung gebracht werden können.

Eine weitergehende Diagnosesicherung durch andere bildgebende Verfahren ist nur bedingt möglich, da diese eine geringere Sensitivität in der Peripherie aufweisen oder invasiv sind (Pulmonalisangiographie). Der Nachweis größerer, nicht okkludierender Emboli durch die CTPA, z.B. Sattelemboli auf der Bifurkation, wäre im Einzelfall jedoch möglich und hilfreich (siehe offene Fragen VI.3.).

Das Alter der Lungenembolie kann ohne Vorbefunde weder durch die V/P-Szintigraphie noch durch die CTPA mit Sicherheit bestimmt werden.

\section{Szintigraphische pulmonale und extrapulmonale Nebenbefunde}

Neben der Lungenembolie können mit der V/P-Szintigraphie noch die COPD, Pneumonie und Linksherzinsuffizienz erkannt werden (13). Leitsymptom der akuten LE ist neben der Tachykardie die Dyspnoe. Häufigste Ursache hierfür ist die Atemwegsobstruktion, erkennbar an einer zentralen Aktivitätdeposition im Aerosol-Ventilationszintigramm und ggf. auch an V/P-match Befunden. Bei einem V/P-reverse-mismatch ist radiologisch eine weitere Klärung zum Ausschluss einer Pneumonie erforderlich, die klinische Bedeutung (funktioneller Shunt) ist im Befundbericht herauszustellen. Der Pseudo-V/P-mismatch darf nicht mit einer LE verwechselt werden, er findet sich relativ häufig mit inhomogener Ausprägung in den Unterfeldern als Hinweis auf eine Peribronchitis. Selten weist er eine Segmentform mit ebensolcher Verschattung im Rö-Bild oder im CT auf. Eine Perfusionsumverteilung von caudal nach cranial, als Perfusionsgradient mit SPECT erfasst, ist Hinweis auf eine kardiale Insuffizienz (57). Weitere Differenzialdiagnosen, insbesondere Bronchial-Ca, siehe Kapitel H. 5. und 6.

\section{Szintigraphische Befunde im Kindesalter}

Die Lungenembolie im Kindesalter ist eine ausgesprochene Rarität, die aber im Einzelfall einen ungünstigen Verlauf nehmen kann $(15,144)$. In der Regel erfahren die Ergebnisse der Lungenszintigraphie im Kindesalter eine Umdeutung. Ein V/P-mismatch ist primär als Gefäßanomalie zu deuten, ein Segment wird nicht mit venösem Blut aus der Pulmonalarterie versorgt, sondern mit arteriellem Blut gewöhnlich direkt aus der Aorta (Sequestration) (77). Ein V/P-match-Befund deutet auf eine Fehlanlage der Bronchien hin (WilliamsCampell Syndrom). Beim Swyer James (MacLeod's) Syndrom, Folge einer Bronchiolitis obliterans im Kindesalter, das gelegentlich erst im Erwachsenenalter erkannt wird (126), zeigt sich eine unilateral erhöhte Transparenz im Rö-Thorax und ein korrespondierender V/P-match-Befund. Die Symptome können einer LE ähneln (126). Eine begleitende LE wird am zusätzlichen V/P-mismatch erkannt (118). Alle genannten Zustände gehen mit einer erhöhten Infektanfälligkeit einher.

\section{Intrapulmonaler Shunt}

Extrapulmonale Aktivität im Perfusionsszintigramm, die sich außerhalb der Lungengrenzen in der Schilddrüse oder in den Nieren findet, kann entweder auf einen Rechts-links-Shunt, auf freies Tc-99m-Pertechnetat oder auf eine vorangegangene andere nuklearmedizinische Untersuchung zurückgehen. Um einen Shunt besser zu erkennen, empfiehlt sich zusätzlich ein Szintigramm des Kopfes (Quantifizierung siehe unten). Außer bei verschiedenen Vitien findet sich ein intrapulmonaler Rechtslinks-Shunt bei Morbus Osler, Morbus Waldenström, Lungenfibrose, a.v. Angiom, Leberzirrhose und CREST-Syndrom. Der Rechts-links-Shunt äußert sich durch extrapulmonale Aktivität vorzugsweise in den gut perfundierten Organen Nieren und Hirn nach intravenöser Applikation von Tc-99m MAA. Zur Quantifizierung ist eine Aufnahme der Nieren oder des Hirns zusätzlich zu den Lungen erforderlich. Hier können die intra- und extrapulmonale Impulse in Beziehung gesetzt werden, verein- 
facht werden die über den Nieren $(25 \%$ des Herzminutenvolumens) bzw. des Hirns (20\% des Herzminutenvolumens) gemessenen Impulse als pars pro toto eingesetzt:

$\frac{\text { Impulse-Nieren x } 4}{\text { Impulse-Nieren x 4 + Impulse-Lungen }} \times 100=$ Shunt (\%)
oder
$\frac{\text { Impulse-Hirn x 5 }}{\text { Impulse-Hirn x 5 + Impulse-Lungen }} \times 100=$ Shunt (\%)

Fehler ergeben sich durch unterschiedliche Absorptionsverhältnisse in den lufthaltigen Lungen und im übrigen Körper sowie durch eventuell freies Pertechnetat in der Tc-99m MAA Präparation. Auf eine Reduktion der MAA-Partikel ist zu achten (Kapitel V.C.).

\section{Befundung bei der Fragestellung chronisch thromboembolische Pul- monale Hypertonie (CTEHP)}

Für die szintigraphische Diagnostik der CTEPH gelten die gleichen Grundsätze wie bei der der akuten LE. SPECT ist der planaren Bildgebung bei dieser Fragestellung überlegen (Sensitivität $63,5 \% \pm 3,1 \%$ versus $42,7 \pm 3,2 \%, \mathrm{p}<0,01)(133)$.

Die chronisch thromboembolische Hypertonie (chronic thromboembolic hypertension [CTEPH]) gilt als seltene, aber schwere Komplikation nach einer oder nach rezidivierenden Lungenembolien. Kennzeichen ist eine narbige Obstruktion der Lungenarterien, die von komplexen Wandveränderungen der pulmonalen Widerstandsgefäße begleitet wird (150). Innerhalb der ersten 2 Jahre nach akuter LE entwickeln zwischen $0,1 \%$ und 9,1\% (69) bzw. im Mittel 5,5\% der Patienten (61) eine CTEPH. Die Inzidenz wird aktuell auf 4 Fälle pro 1 Million Personen/Jahr (43) und die Prävalenz auf 3-30 Fälle pro 1 Million Personen/Jahr geschätzt (62). Klinische Zeichen oder Symptome sind in den frühen Stadien uncharakteristisch, die mediane Zeit vom Beginn der Symptomatik bis zur Diagnose beträgt 14 Monate oder länger (103). Bei Patienten mit persistierender Dyspnoe nach dreimonatiger effektiver Antikoagulation einer LE sollte ein eine CTEPH-Diagnostik durchgeführt werden. Ein generelles Screening asymptomatischer
Patienten nach LE oder tiefer Venenthrombose wird dagegen nicht empfohlen.

Unterschieden werden kann eine mehr zentral lokalisierte CTEPH, die einer operativen Behandlung durch Embolektomie zugänglich ist, von einer peripheren CTEPH. Bei zentraler CTEPH ist V/P-planar der CTPA nur leicht überlegen (Sensitivität $100 \%$ vs. $92,2 \%$ ) (39). V/P-SPECT erzielt bessere Ergebnisse, die Sensitivität beim Nachweis verschlossener Segmente beträgt $62 \pm 4,1 \%$ für V/P-SPECT und $47,8 \pm 2,9 \%$ für die CTPA $(133,134)$. In einem Patientenkollektiv mit sowohl zentraler als auch peripherer CTEPH erwies sich selbst V/P-planar deutlich sensitiver als die CTPA: Sensitivität 96\%-97,4\% und Spezifität $90 \%-95 \%$ vs. Sensitivität $51 \%$ und Spezifität 99\% (139). Perfusionsdefekte, die mit der Dual-Energie CT (DECT) nachweisbar sind, weisen auf der Segmentebene eine nur mäßige Übereinstimmung $(\mathrm{k}=0,44)$ mit denen der V/P-Szintigraphie auf, die Kongruenz mit der CTPA ist noch geringer $(k=0,09-0,31)(20)$. Im Algorithmus der Abklärung der pulmonalen Hypertonie hat daher die Ventilations- und Perfusions-SPECT einen zentralen Stellenwert $(96,149)$ und kann auch bei der Identifikation operabler Patienten hilfreich sein (34).

\section{J. Qualitätssicherung}

Die Präparation der Radiopharmaka erfolgt gemäß den Vorschriften des Herstellers. In Deutschland und der Schweiz sind regelmäßige Qualitätskontrollen vorgeschrieben, es soll hierzu das vom Hersteller empfohlene Verfahren zur Anwendung kommen. Wird im Perfusionsszintigramm eine Anreicherung in der Schilddrüse und im Magen infolge von freiem ${ }^{99 \mathrm{~m}} \mathrm{Tc}-\mathrm{Per}-$ technetat beobachtet, ist eine Überprüfung der radiochemischen Reinheit jeder Markierung der gesamten Charge erforderlich. Eine intensive zentrale Aktivitätsdeposition im Aerosol-Ventilationszintigramm kann bei ultrafeinem Trockenaerosol unter anderem auch Hinweis auf eine fehlerhafte Zubereitung sein: Wartezeit bis zur Inhalation $>10$ Min. oder mehrfaches Eindampfen.

\section{K. Fehlerquellen}

Ventilations- und Perfusionsszintigraphie werden zu unterschiedlichen Zeitpunkten durchgeführt. Im dazwischen liegenden Zeitraum können Änderungen in der regionalen Ventilation und Perfusion eintreten. Ebenso wird in der Regel Radioaerosol in aufrechter Position inhaliert und Tc-99m-MAA in Rückenlage injiziert. Die in unterschiedlicher Position fixierte Verteilung der Radiopharmaka kann ebenfalls die Vergleichbarkeit beeinträchtigen.

Die Applikation von Tc-99m-MAA über einen zentralen Zugang kann eine unzureichende Durchmischung der Aktivität in der Pulmonalarterie zur Folge haben, insbesondere dann, wenn die Aktivität durch einen Katheter direkt in die Pulmonalarterie appliziert wird.

Eine Schräglage des Patienten beeinflusst die regionale Perfusion mehr als die regionale Ventilation. Bei Applikation von Tc-99m-MAA in dieser Position kann ein großflächiges mismatch-Verteilungsmuster resultieren.

„Hot spots“ im Perfusionsszintigramm treten durch Blutkoagel auf, die sich entweder in der Spritze selbst bilden (kein Blut aspirieren, dünne Kanüle verwenden) oder wenn die Injektion durch einen schlecht gespülten Katheter erfolgte.

\section{Vorbehaltserklärung}

Die Deutsche Gesellschaft für Nuklearmedizin gibt Leitlinien heraus, um die Anwendung kostengünstiger Untersuchungsverfahren und Behandlungsmethoden in der Nuklearmedizin zu fördern. Diese Art von Empfehlungen gilt nicht für alle Gegebenheiten in der Praxis. Die Leitlinien sollen nicht den Anspruch erheben, dass sie alle in Frage kommenden Verfahren enthielten oder, als ob sie Methoden, die zum gleichen Ergebnis führen, ausschließen würden. Das Leistungsspektrum für Patienten in einer speziellen Praxis kann ganz anders aussehen als für Patienten, die in einem mehr allgemein medizinischen Rahmen untersucht werden. Ob ein Untersuchungsverfahren angemessen ist, hängt zum Teil von der Prävalenz der Erkrankung in der Patientenpopulation ab. Au- 
ßerdem können sich die zur Verfügung stehenden Möglichkeiten bei verschiedenen medizinischen Einrichtungen sehr unterscheiden. Aus diesen Gründen dürfen Leitlinien nicht starr angewendet werden.

Fortschritte in der Medizin vollziehen sich schnell. Deshalb muss bei der Benutzung einer Leitlinie auf ihr Entstehungsdatum geachtet werden.

\section{Offene Fragen in der der Emboliediagnostik}

\section{Schwellenwerte zur Vermeidung einer Überdiagnostik}

In der Behandlung der akuten LE zeichnen sich bei kreislaufstabilen Patienten zwei Entwicklungen ab: ambulante Therapie (115) versus stationäre Beobachtung (primär ohne Therapie) (146). Im ersteren Fall wird unabhängig vom klinischen Schweregrad bei nahezu sämtlichen Patienten $(96,8 \%)$ eine Therapie eingeleitet, sobald eine LE mit hoher Sicherheit diagnostiziert wird (in der CTPA Füllungsdefekt auf der Segmentebene oder darüber). Befunde auf der Subsegmentebene werden nur dann berücksichtigt, wenn sonographisch zusätzlich eine tiefe Beinvenenthrombose gesichert wird oder die ergänzend durchgeführte Szintigraphie einen High-Probability-Befund gemäß PIOPED-Kriterien zeigt (115). In diesem Kontext wird auf eine erhöhte Nachweisrate kleiner Emboli und eine möglicherweise daraus resultierende Überdiagnostik mit V/P-SPECT hingewie$\operatorname{sen}(82)$.

Um dem vorzubeugen und um die Diagnose LE $\mathrm{zu}$ rechtfertigen, wird bei V/P-SPECT in Übereinstimmung mit der Europäischen Leitlinie $(7,8)$ ein unterer Schwellenwert festgelegt, der einen V/P-mismatch in einem Segment oder in mindestens zwei Subsegmenten, entweder räumlich getrennt oder zusammenliegend, entsprechen soll. Unter diesen Voraussetzungen wird ein ausgeglichenes Verhältnis zwischen Sensitivität und Spezifität von jeweils $92 \%$ erzielt (72). Darüber hinaus wurde der empirisch ermittelte Schwellenwert nicht validiert. Der Verlust an Sensitivität durch die Schwelle beträgt absolut ca. $3 \%$ (35). Bei diesen Patienten wird die Diagnose LE unterdrückt und klinisch nicht verwertet. Ziel war es, trotz höherer Sensitivität von V/P-SPECT auf der Subsegmentebene die Zahl therapierelevanter Befunde gegenüber V/P-planar nicht übermäßig ansteigen zu lassen.

Werden die Patienten im zunehmenden Maße (vorerst nur in den USA) nur noch stationär beobachtet $(91,146)$, so wird ein oberer Schwellenwert erforderlich. Hochrisikopatienten (>80 Jahre, Herzinsuffizienz, chronische Lungenerkrankung, Nierenoder Lebererkrankung, hohes Blutungsrisiko, Karzinom oder Thrombombolyse/Embolektomie erforderlich) sollten ausgenommen werden (91).

Sollte sich eine risikoorientierte zweistufige Behandlungsstrategie (Stufe 1: Beobachtung, Stufe 2: Anikoagulation stationär oder ambulant) in Zukunft durchsetzen, so würden alle isolierten Befunde auf der Subsegmentebene in die Niedrigrisikogruppe eingeordnet und als LE mitgeteilt ohne eine Übertherapie zu induzieren. Ohne Unterdrückung der Diagnose LE würde die bisher eventuell erforderliche Umdeutung eines Nebenbefunds in der CTPA zur behandlungsbedürftigen Alternativdiagnose vermieden, die Vorgehensweise bei wiederkehrender Symptomatik sofort in die richtigen Bahnen gelenkt und das diagnostische Potenzial der V/P-Szintigraphie voll genutzt.

\section{Klinische Bedeutung teil- und nichtokkludierender Emboli}

Emboli verschließen die Endarterie quantitativ unterschiedlich, mit der computerunterstützten Auswertung der CTPA zeigen sich Okklusionsgrade von 5-100\% (155). Zentrale Emboli, die mit der CTPA im Truncus pulmonalis und in der Gefäßbifurkation (Sattelembolus) gesehen werden, sind grundsätzlich nichtokkludierend und szintigraphisch nicht oder nur schwer erkennbar. Ein Teilverschluss der linken oder rechten Pulmonalarterie bleibt in der Regel ebenfalls unsichtbar, der komplette Verschluss einer Pulmonalarterie mit vollständigem Perfusionsausfall einer Lunge ist bei normotensiven Patienten eine Rarität. Auch bei wandständigen Emboli in einer Lappenarterie kann das Perfusionsszintigramm normal sein.
Zur Häufigkeit zentraler Emboli, die mit der CTPA bei normotensiven Patienten nachgewiesen werden, liegen unterschiedliche Angaben vor: Bei jüngeren Patienten $6 \%$ (73), bei Erwachsenen reicht die Spanne von $36,5 \%$ (127) bis $60 \%$ (145). Ob der zentrale Embolus ein unabhängiger Prädiktor von Mortalität oder klinischer Verschlechterung ist, wird sowohl bejaht (145) als auch verneint (127).

\section{Postoperative Lungen- funktion}

Als quantitatives Maß für die globale ventilatorische Ausgangskapazität hat sich das forcierte Einsekundenvolumen FEV1 bewährt, das sowohl obstruktive als auch restriktive Insuffizienzen aufzeigt. Eine individuelle Voraussage der postoperativen Lungenfunktion empfiehlt die Deutsche Gesellschaft für Pneumologie und die Deutsche Gesellschaft für Thorax-, Herz- und Gefäßchirurgie bei folgenden präoperativen Grenzwerten: $<2,5$ Liter vor Pneumektomie, $<1,75$ Liter vor Lobektomie und $<1,5$ Liter vor Segmentresektion. Die postoperative Lungenfunktion nach Pneumektomie wird berechnet, indem das präoperativ bestimmte FEV1 mit dem prozentualem Funktionswert der verbleibenden Lunge multipliziert wird und dieser Wert durch 100 geteilt wird. Im Falle einer Lobektomie oder Segmentresektion muss der verbleibende Funktionsanteil der betroffenen Lunge mit berücksichtigt werden, was bei planarer Aufnahmetechnik nur durch Näherung in $25 \%$-Schritten geht. Das berechnete postoperative FEV1 soll $>0,8$ bis 1,0 Liter betragen.

Vor einer Lungenresektion ist eine dreidimensionale Darstellung der präoperativen Lungenperfusion oder -ventilation von Vorteil. Bei Anwendung von SPECT/CT erweist sich die Korrelation der postoperativen Ventilationgröße FEV1 sowohl mit der regionalen Restperfusion (Tc-99m MAA) als auch mit der regionalen Restventilation $(\mathrm{Kr}-81 \mathrm{~m})$ als gleichwertig (94). SPECT/CT erlaubt eine genauere Abgrenzung der ROI und damit bessere Korrelation mit dem postoperativem FEV1 ( $\mathrm{R}=0,993$ vs. $\mathrm{R}=0,890$ ) (135), wobei SPECT/CT auch für die Schwächungskor- 
rektur eingesetzt werden sollte (138). Beim Vergleich Perfusion planar mit Perfusion SPECT/CT finden sich Abweichungen bis $10,9 \%$ im linken Oberlappen sowie bis $22,9 \%$ im rechten Oberlappen (66). Die regionale Ventilationsdarstellung mit Ga-68 Nanopartikel PET korreliert eng mit der vom CT abgeleiteten Ventilation (25), im Einzelfall können jedoch Abweichungen zum V PET/CT auftreten (63).

Sofern sowohl der seitengetrennte Perfusions- als auch Ventilationsanteil mit SPECT oder mit SPECT/CT bestimmt wird, ist bei Diskrepanzen der mit der Ventilationsszintigraphie gewonnene Voraussagewert der validere.

\section{Literatur}

\section{Uncategorized References}

1. Angriman F, Ferreyro BL, Posadas-Martinez ML et al. Wells Score and Poor Outcomes Among Adult Patients With Subsegmental Pulmonary Embolism: A Cohort Study. Clin Appl Thromb Hemost 2015; 21: 539-545.

2. Astani SA, Davis LC, Harkness BA et al. Detection of pulmonary embolism during pregnancy: comparing radiation doses of CTPA and pulmonary scintigraphy. Nucl Med Commun 2014; 35: 704-711.

3. Bach AG, Restrepo CS, Abbas J et al. Imaging of nonthrombotic pulmonary embolism: biological materials, nonbiological materials, and foreign bodies. Eur J Radiol 2013; 82: e120-141.

4. Bach AG, Taute BM, Baasai N et al. 30-Day Mortality in Acute Pulmonary Embolism: Prognostic Value of Clinical Scores and Anamnestic Features. PLoS One 2016; 11: e0148728.

5. Bajc M, Maffioli L, Miniati M. Good clinical practice in pulmonary embolism diagnosis: where do we stand today? Eur J Nucl Med Mol Imaging 2014; 41: 333-336.

6. Bajc M, Miniati M, Jogi J et al. Perfusion SPECT in patients with suspected pulmonary embolism. Eur J Nucl Med Mol Imaging 2013; 40: 1432-1437.

7. Bajc M, Neilly JB, Miniati M et al. EANM guidelines for ventilation/perfusion scintigraphy : Part 2. Algorithms and clinical considerations for diagnosis of pulmonary emboli with $\mathrm{V} / \mathrm{P}(\mathrm{SPECT})$ and MDCT. Eur J Nucl Med Mol Imaging 2009; 36: $1528-1538$

8. Bajc M, Neilly JB, Miniati M et al. EANM guidelines for ventilation/perfusion scintigraphy : Part 1. Pulmonary imaging with ventilation/perfusion single photon emission tomography. Eur J Nucl Med Mol Imaging 2009; 36: 1356-1370.

9. Bajc M, Olsson B, Gottsater A et al. V/P SPECT as a diagnostic tool for pregnant women with suspected pulmonary embolism. Eur J Nucl Med Mol Imaging 2015; 42: 1325-1330.

10. Bajc M, Olsson CG, Olsson B et al. Diagnostic evaluation of planar and tomographic venti- lation/perfusion lung images in patients with suspected pulmonary emboli. Clin Physiol Funct Imaging 2004; 24: 249-256.

11. Baysson H, Journy N, Roue T et al. [Exposure to CT scans in childhood and long-term cancer risk: A review of epidemiological studies]. Bull Cancer 2016; 103: 190-198.

12. Becattini C, Agnelli G, Lankeit $M$ et al. Acute pulmonary embolism: mortality prediction by the 2014 European Society of Cardiology risk stratification model. Eur Respir J 2016; 48: 780-786.

13. Begic A, Opankovic E, Cukic V et al. Ancillary findings assessed by ventilation/perfusion tomography. Impact and clinical outcome in patients with suspected pulmonary embolism. Nuklearmedizin 2015; 54: 223-230.

14. Boc $\mathrm{A}$, Vene $\mathrm{N}$, Kosmelj $\mathrm{K}$ et al. Impact of Asymptomatic Pulmonary Embolism on the Long-Term Prognosis of Patients with Deep Venous Thrombosis. Semin Thromb Hemost 2017; 43: 24-29.

15. Byard RW. Fatal embolic events in childhood. J Forensic Leg Med 2013; 20: 1-5.

16. Carrier M, Righini M, Wells PS et al. Subsegmental pulmonary embolism diagnosed by computed tomography: incidence and clinical implications. A systematic review and meta-analysis of the management outcome studies. J Thromb Haemost 2010; 8: 1716-1722.

17. Castellani M, Vanoli M, Cali G et al. Ventilationperfusion lung scan for the detection of pulmonary involvement in Takayasu's arteritis. Eur J Nucl Med 2001; 28: 1801-1805.

18. Cook JV, Kyriou J. Radiation from CT and perfusion scanning in pregnancy. BMJ 2005; 331: 350.

19. den Exter PL, Jimenez D, Kroft LJ et al. Outcome of incidentally diagnosed pulmonary embolism in patients with malignancy. Curr Opin Pulm Med 2012; 18: 399-405.

20. Dournes G, Verdier D, Montaudon $\mathrm{M}$ et al. Dual-energy CT perfusion and angiography in chronic thromboembolic pulmonary hypertension: diagnostic accuracy and concordance with radionuclide scintigraphy. Eur Radiol 2014; 24: 42-51.

21. EANM. Dosage Card. http://eanmorg/publi cations/dosage_calculatorphp?navId=285:

EANM Executive Office; 2016.

22. Easther S, Langdana F, Beasley R et al. The diagnostic role of ventilation/perfusion scans versus computed tomography pulmonary angiography in obstetric patients investigated for pulmonary embolism at Wellington Hospital from 2010 to 2012. N Z Med J 2016; 129: 62-68.

23. Einstein AJ, Henzlova MJ, Rajagopalan S. Estimating risk of cancer associated with radiation exposure from 64-slice computed tomography coronary angiography. JAMA 2007; 298: 317-323.

24. Elbakri IA, Kirkpatrick DC. Survey of clinical doses from computed tomography examinations in the Canadian province of Manitoba. Radiat Prot Dosimetry 2013; 157: 525-535.

25. Eslick EM, Bailey DL, Harris B et al. Measurement of preoperative lobar lung function with computed tomography ventilation imaging: progress towards rapid stratification of lung cancer lobectomy patients with abnormal lung function. Eur J Cardiothorac Surg 2016; 49: 1075-1082.

26. Fanikos J, Rao A, Seger AC et al. Hospital costs of acute pulmonary embolism. Am J Med 2013; 126: 127-132.

27. Furlan A, Aghayev A, Chang CC et al. Shortterm mortality in acute pulmonary embolism: clot burden and signs of right heart dysfunction at CT pulmonary angiography. Radiology 2012; 265: 283-293.

28. Garcia-Fuster MJ, Fabia MJ, Furio E et al. Should we look for silent pulmonary embolism in patients with deep venous thrombosis? BMC Cardiovasc Disord 2014; 14: 178.

29. Geraci G, Lo Iacono G, Lo Nigro C et al. Asymptomatic bone cement pulmonary embolism after vertebroplasty: case report and literature review. Case Rep Surg 2013; 2013: 591432.

30. Ghazvinian R, Gottsater A, Elf J. Is it safe to withhold long-term anticoagulation therapy in patients with small pulmonary emboli diagnosed by SPECT scintigraphy? Thromb J 2016 14: 12 .

31. Glaser JE, Chamarthy M, Haramati LB et al. Successful and safe implementation of a trinary interpretation and reporting strategy for V/Q lung scintigraphy. J Nucl Med 2011; 52: 1508-1512.

32. Goodman LR, Lipchik RJ, Kuzo RS et al. Subsequent pulmonary embolism: risk after a negative helical CT pulmonary angiogram--prospective comparison with scintigraphy. Radiology 2000; 215: 535-542

33. Gradistanac T, Wittekind C. [Autopsy as a tool for quality assurance: Leipzig]. Pathologe 2011; 32 Suppl 2: 287-291.

34. Grgic A, Miodek F, Schafers HJ et al. Assessment of operability by means of CTPA and perfusion SPECT in patients with chronic thromboembolic pulmonary hypertension. Acta Radiol 2016; 57: 33-40

35. Gruning T, Drake BE, Farrell SL et al. Three-year clinical experience with VQ SPECT for diagnosing pulmonary embolism: diagnostic performance. Clin Imaging 2014; 38: 831-835.

36. Gutte H, Mortensen J, Jensen CV et al. Detection of pulmonary embolism with combined ventilation-perfusion SPECT and low-dose CT: headto-head comparison with multidetector CT angiography. J Nucl Med 2009; 50: 1987-1992.

37. Hammer GP, Auvinen A, De Stavola BL et al. Mortality from cancer and other causes in commercial airline crews: a joint analysis of cohorts from 10 countries. Occup Environ Med 2014; 71: 313-322.

38. Harris B, Bailey D, Miles S et al. Objective analysis of tomographic ventilation-perfusion scintigraphy in pulmonary embolism. Am J Respir Crit Care Med 2007; 175: 1173-1180.

39. He J, Fang W, Lv B et al. Diagnosis of chronic thromboembolic pulmonary hypertension: comparison of ventilation/perfusion scanning and multidetector computed tomography pulmonary angiography with pulmonary angiography. Nucl Med Commun 2012; 33: 459-463.

40. He J, Wang F, Dai HJ et al. Chinese multi-center study of lung scintigraphy and CT pulmonary angiography for the diagnosis of pulmonary embolism. Int J Cardiovasc Imaging 2012; 28 : 1799-1805. 
41. Hee L, Ng AC, Huang J et al. The contribution of cardiovascular mortality to long term outcomes in a relatively young demographic following acute pulmonary embolism: a validation study. Int J Cardiol 2015; 199: 13-17.

42. Hess S, Frary EC, Gerke O et al. State-of-the-Art Imaging in Pulmonary Embolism: Ventilation/ Perfusion Single-Photon Emission Computed Tomography versus Computed Tomography Angiography - Controversies, Results, and Recommendations from a Systematic Review. Semin Thromb Hemost 2016; 42: 833-845.

43. Hoeper MM, Humbert M, Souza R et al. A global view of pulmonary hypertension. Lancet Respir Med 2016; 4: 306-322.

44. Hoffmann B, Gross CR, Jockel KH et al. Trends in mortality of pulmonary embolism--an international comparison. Thromb Res 2010; 125: 303-308.

45. Homolka P, Leithner R, Billinger J et al. [Results of the Austrian CT dose study 2010: typical ef fective doses of the most frequent CT examinations]. Z Med Phys 2014; 24: 224-230.

46. Hurwitz LM, Reiman RE, Yoshizumi TT et al. Radiation dose from contemporary cardiothoracic multidetector CT protocols with an anthropomorphic female phantom: implications for cancer induction. Radiology 2007; 245: 742-750.

47. Hurwitz LM, Yoshizumi T, Reiman RE et al Radiation dose to the fetus from body MDCT during early gestation. AJR Am J Roentgenol 2006; 186: 871-876.

48. Ibanez-Bravo S, Banzo I, Quirce R et al. Ventilation/Perfusion SPECT lung scintigraphy and computed tomography pulmonary angiography in patients with clinical suspicion of pulmonary embolism. Rev Esp Med Nucl Imagen Mol 2016; 35: 215-220.

49. ICRP. Radiation dose to patients from radiopharmaceuticals. ICRP Publication 53). Ann ICRP 1988; 18.

50. ICRP. Radiation dose to patients from radiopharmaceuticals (Adenum to ICRP Publication 53). ICRP Publication 80). Ann ICRP 1998; 28.

51. Ikesaka R, Carrier M. Clinical significance and management of subsegmental pulmonary embolism. J Thromb Thrombolysis 2015; 39: 311-314.

52. Inayat F, Cheema AR, Virk HU et al. Nonthrombotic Pulmonary Embolism: A Potential Complication of Polyacrylamide Hydrogel Cosmetic Injection. Case Rep Med 2016; 2016: 1397434.

53. Investigators P. Value of the ventilation/perfusion scan in acute pulmonary embolism. Results of the prospective investigation of pulmonary embolism diagnosis (PIOPED). JAMA 1990; 263: 2753-2759.

54. Janata K, Holzer M, Domanovits H et al. Mortality of patients with pulmonary embolism. Wien Klin Wochenschr 2002; 114: 766-772.

55. Jogi J, Jonson B, Ekberg M et al. Ventilation-perfusion SPECT with 99mTc-DTPA versus Technegas: a head-to-head study in obstructive and nonobstructive disease. J Nucl Med 2010; 51: 735-741.

56. Jogi J, Markstad H, Tufvesson E et al. The added value of hybrid ventilation/perfusion SPECT/CT in patients with stable COPD or apparently healthy smokers. Cancer-suspected CT findings in the lungs are common when hybrid imaging is used. Int J Chron Obstruct Pulmon Dis 2015; 10: $25-30$.

57. Jogi J, Palmer J, Jonson B et al. Heart failure diagnostics based on ventilation/perfusion single photon emission computed tomography pattern and quantitative perfusion gradients. Nucl Med Commun 2008; 29: 666-673.

58. Jonsson KO, Agnarsson UT, Danielsen $\mathrm{R}$ et al. [Pulmonary embolism at Landspitali, the National University Hospital of Iceland 2005-2007 - incidence, clinical manifestations, risk factors and outcome]. Laeknabladid 2013; 99: 11-15.

59. Kan Y, Yuan L, Meeks JK et al. The accuracy of V/Q SPECT in the diagnosis of pulmonary embolism: a meta-analysis. Acta Radiol 2015; 56: 565-572.

60. Kawakami K, Iwamura A, Goto E et al. [Kinetics and clinical application of $99 \mathrm{mTc}$-technegas]. Kaku Igaku 1990; 27: 725-733

61. Kayaalp I, Varol Y, Cimen P et al. The incidence of chronic thromboembolic pulmonary hypertension secondary to acute pulmonary thromboembolism. Tuberk Toraks 2014; 62: 199-206.

62. Kim NH, Delcroix M, Jenkins DP et al. Chronic thromboembolic pulmonary hypertension. J Am Coll Cardiol 2013; 62: D92-99.

63. Kipritidis J, Siva S, Hofman MS et al. Validating and improving CT ventilation imaging by correlating with ventilation 4D-PET/CT using $68 \mathrm{Ga}$ labeled nanoparticles. Med Phys 2014; 41: 011910.

64. Klok FA, Mos IC, Nijkeuter M et al. Simplification of the revised Geneva score for assessing clinical probability of pulmonary embolism. Arch Intern Med 2008; 168: 2131-2136.

65. Klok FA, van Kralingen KW, van Dijk AP et al. Quality of life in long-term survivors of acute pulmonary embolism. Chest 2010; 138: 1432-1440.

66. Knollmann D, Meyer A, Noack F et al. Preoperative assessment of relative pulmonary lobar perfusion fraction in lung cancer patients. A rather simple three-dimensional CT-based vs. planar image-derived quantification. Nuklearmedizin 2015; 54: 178-182.

67. Konstantinides SV, Vicaut E, Danays T et al. Impact of Thrombolytic Therapy on the LongTerm Outcome of Intermediate-Risk Pulmonary Embolism. J Am Coll Cardiol 2017; 69: 1536-1544.

68. Kurt J, Uhländer J, Schwarzenböck SM et al. Added value of automated detection of match and mismatch-defects in the diagnosis of pulmonary embolism. Nuklearmedizin 2015; 54: A133.

69. Lang IM, Simonneau G, Pepke-Zaba JW et al. Factors associated with diagnosis and operability of chronic thromboembolic pulmonary hypertension. A case-control study. Thromb Haemost 2013; 110: 83-91.

70. Laribi S, Aouba A, Resche-Rigon M et al. Trends in death attributed to myocardial infarction, heart failure and pulmonary embolism in Europe and Canada over the last decade. QJM 2014; 107: 813-820.

71. Le Gal G, Righini M, Roy PM et al. Prediction of pulmonary embolism in the emergency depart- ment: the revised Geneva score. Ann Intern Med 2006; 144: 165-171.

72. Le Roux PY, Robin P, Delluc A et al. V/Q SPECT interpretation for pulmonary embolism diagnosis: which criteria to use? J Nucl Med 2013; 54: 1077-1081.

73. Lee EY, Neuman MI, Lee NJ et al. Pulmonary embolism detected by pulmonary MDCT angiography in older children and young adults: risk factor assessment. AJR Am J Roentgenol 2012; 198: 1431-1437.

74. Leung AN, Bull TM, Jaeschke R et al. American Thoracic Society documents: an official American Thoracic Society/Society of Thoracic Radiology Clinical Practice Guideline--Evaluation of Suspected Pulmonary Embolism in Pregnancy. Radiology 2012; 262: 635-646.

75. Leuschner G, Wenter V, Milger $\mathrm{K}$ et al. Suspected pulmonary embolism in patients with pulmonary fibrosis: Discordance between ventilation/perfusion SPECT and CT pulmonary angiography. Respirology 2016; 21: 1081-1087.

76. Ling IT, Naqvi HA, Siew TK et al. SPECT ventilation perfusion scanning with the addition of low-dose CT for the investigation of suspected pulmonary embolism. Intern Med J 2012; 42: 1257-1261.

77. Loewy J, O’Brodovich H, Coates G. Ventilation scintigraphy with submicronic radioaerosol as an adjunct in the diagnosis of congenital lobar emphysema. J Nucl Med 1987; 28: 1213-1217.

78. Long B, Koyfman A. Best Clinical Practice: Controversies in Outpatient Management of Acute Pulmonary Embolism. J Emerg Med 2017; 52: 668-679.

79. MacGregor K, Li I, Dowdell T et al. Identifying Institutional Diagnostic Reference Levels for CT with Radiation Dose Index Monitoring Software. Radiology 2015; 276: 507-517.

80. Mathews JD, Forsythe AV, Brady Z et al. Cancer risk in 680,000 people exposed to computed tomography scans in childhood or adolescence: data linkage study of 11 million Australians. BMJ 2013; 346: f2360.

81. Mazurek A, Dziuk M, Witkowska-Patena E et al. The Utility of Hybrid SPECT/CT Lung Perfusion Scintigraphy in Pulmonary Embolism Diagnosis. Respiration 2015; 90: 393-401.

82. Metter D, Tulchinsky M, Freeman LM. Current Status of Ventilation-Perfusion Scintigraphy for Suspected Pulmonary Embolism. AJR Am J Roentgenol 2017; 208: 489-494.

83. Miller WT, Jr., Marinari LA, Barbosa E, Jr. et al. Small pulmonary artery defects are not reliable indicators of pulmonary embolism. Ann Am Thorac Soc 2015; 12: 1022-1029.

84. Miniati M, Bottai M, Monti S et al. Simple and accurate prediction of the clinical probability of pulmonary embolism. Am J Respir Crit Care Med 2008; 178: 290-294.

85. Miniati M, Pistolesi M, Marini C et al. Value of perfusion lung scan in the diagnosis of pulmonary embolism: results of the Prospective Investigative Study of Acute Pulmonary Embolism Diagnosis (PISA-PED). Am J Respir Crit Care Med 1996; 154: 1387-1393.

86. Monaghan P, Provan I, Murray C et al. An improved radionuclide technique for the detection 
of altered pulmonary permeability. J Nucl Med 1991; 32: 1945-1949.

87. Moores LK, Jackson WL, Jr., Shorr AF et al. Meta-analysis: outcomes in patients with suspected pulmonary embolism managed with computed tomographic pulmonary angiography. Ann Intern Med 2004; 141: 866-874.

88. Morley NC, Muir KC, Mirsadraee S et al. Ten years of imaging for pulmonary embolism: too many scans or the tip of an iceberg? Clin Radiol 2015; 70: 1370-1375.

89. Moser KM, Fedullo PF, LitteJohn JK et al. Frequent asymptomatic pulmonary embolism in patients with deep venous thrombosis. JAMA 1994; 271: 223-225.

90. Ng AC, Chung T, Yong AS et al. Long-term cardiovascular and noncardiovascular mortality of 1023 patients with confirmed acute pulmonary embolism. Circ Cardiovasc Qual Outcomes 2011; 4: 122-128.

91. Nguyen E, Coleman CI, Peacock WF et al. Observation management of pulmonary embolism and agreement with claims-based and clinical risk stratification criteria in United States patients: a retrospective analysis. BMC Pulm Med 2017; 17: 37.

92. Nielsen HK, Husted SE, Krusell LR et al. Silent pulmonary embolism in patients with deep venous thrombosis. Incidence and fate in a randomized, controlled trial of anticoagulation versus no anticoagulation. J Intern Med 1994; 235: 457-461.

93. Nijkeuter M, Geleijns J, De Roos A et al. Diagnosing pulmonary embolism in pregnancy: rationalizing fetal radiation exposure in radiological procedures. J Thromb Haemost 2004; 2: 1857-1858.

94. Ohno Y, Koyama H, Takenaka D et al. Coregistered ventilation and perfusion SPECT using krypton-81m and Tc-99m-labeled macroaggregated albumin with multislice CT utility for prediction of postoperative lung function in nonsmall cell lung cancer patients. Acad Radiol 2007; 14: 830-838.

95. Olie V, Fuhrman C, Chin F et al. Time trends in pulmonary embolism mortality in France, 2000-2010. Thromb Res 2015; 135: 334-338.

96. Olsson KM, Meyer B, Hinrichs J et al. Chronic thromboembolic pulmonary hypertension. Dtsch Arztebl Int 2014; 111: 856-862.

97. Palmowski K, Oltmanns U, Kreuter M et al. Diagnosis of pulmonary embolism: conventional ventilation/perfusion SPECT is superior to the combination of perfusion SPECT and nonenhanced CT. Respiration 2014; 88: 291-297.

98. Parent F, Maitre S, Meyer G et al. Diagnostic value of $\mathrm{D}$-dimer in patients with suspected pulmonary embolism: results from a multicentre outcome study. Thromb Res 2007; 120: 195-200.

99. Parker JA, Coleman RE, Siegel BA et al. Procedure guideline for lung scintigraphy: 1.0. Society of Nuclear Medicine. J Nucl Med 1996; 37: 1906-1910.

100. Partsch H, Oburger K, Mostbeck A et al. Frequency of pulmonary embolism in ambulant patients with pelvic vein thrombosis: a prospective study. J Vasc Surg 1992; 16: 715-722.

101. Patel N, Patel NJ, Agnihotri K et al. Utilization of catheter-directed thrombolysis in pulmonary embolism and outcome difference between systemic thrombolysis and catheter-directed thrombolysis. Catheter Cardiovasc Interv 2015; 86: 1219-1227.

102. Pearce MS, Salotti JA, Little MP et al. Radiation exposure from CT scans in childhood and subsequent risk of leukaemia and brain tumours: a retrospective cohort study. Lancet 2012; 380: 499-505.

103. Pepke-Zaba J, Delcroix M, Lang I et al. Chronic thromboembolic pulmonary hypertension (CTEPH): results from an international prospective registry. Circulation 2011; 124: 1973-1981.

104. Perisinakis K, Seimenis I, Tzedakis A et al. Perfusion scintigraphy versus 256-slice CT angiography in pregnant patients suspected of pulmonary embolism: comparison of radiation risks. J Nucl Med 2014; 55: 1273-1280.

105. Pesavento R, de Conti G, Minotto I et al. The value of 64-detector row computed tomography for the exclusion of pulmonary embolism. Thromb Haemost 2011; 105: 901-907.

106. Phillips JJ, Straiton J, Staff RT. Planar and SPECT ventilation/perfusion imaging and computed tomography for the diagnosis of pulmonary embolism: A systematic review and meta-analysis of the literature, and cost and dose comparison. Eur J Radiol 2015; 84: 1392-1400.

107. Putter C, von Beckerath O, Sobik HM et al. Prescription of enoxaparin is associated with decreasing pulmonary embolism mortality rate in Germany. J Thromb Thrombolysis 2015; 40: 468-473.

108. Raskob GE. Importance of subsegmental pulmonary embolism. Blood 2013; 122: 1094-1095.

109. Reinartz P, Kaiser HJ, Wildberger JE et al. SPECT imaging in the diagnosis of pulmonary embolism: automated detection of match and mismatch defects by means of image-processing techniques. J Nucl Med 2006; 47: 968-973.

110. Reinartz P, Wildberger JE, Schaefer W et al. Tomographic imaging in the diagnosis of pulmonary embolism: a comparison between V/Q lung scintigraphy in SPECT technique and multislice spiral CT. J Nucl Med 2004; 45: 1501-1508.

111. Reissig A, Haase U, Schulze E et al. [Diagnosis and therapy of pulmonary embolism prior to death]. Dtsch Med Wochenschr 2010; 135: 1477-1483.

112. Reissig A, Kroegel C. [Therapeutic approaches to acute pulmonary embolism]. Internist (Berl) 2004; 45: 540-548.

113. Revel MP, Fitton I, Audureau E et al. Breast dose reduction options during thoracic $\mathrm{CT}$ : influence of breast thickness. AJR Am J Roentgenol 2015; 204: W421-428.

114. Righini M, Van Es J, Den Exter PL et al. Age-adjusted D-dimer cutoff levels to rule out pulmonary embolism: the ADJUST-PE study. JAMA 2014; 311: 1117-1124.

115. Roy PM, Corsi DJ, Carrier M et al. Net clinical benefit of hospitalization versus outpatient management of patients with acute pulmonary embolism. J Thromb Haemost 2017; 15: 685-694.

116. Roy PM, Meyer G, Vielle B et al. Appropriateness of diagnostic management and outcomes of suspected pulmonary embolism. Ann Intern Med 2006; 144: 157-164.
117. Sabarudin A, Mustafa Z, Nassir KM et al. Radiation dose reduction in thoracic and abdomenpelvic CT using tube current modulation: a phantom study. J Appl Clin Med Phys 2014; 16: 5135.

118. Sager S, Asa S, Akyel R et al. Regional ventilation/perfusion mismatch pattern in patient with Swyer James (MacLeod's) syndrome. J Res Med Sci 2014; 19: 904-906.

119. Sanchez O, Helley D, Couchon S et al. Perfusion defects after pulmonary embolism: risk factors and clinical significance. J Thromb Haemost 2010; 8: 1248-1255.

120. Scarvelis D, Anderson J, Davis L et al. Hospital mortality due to pulmonary embolism and an evaluation of the usefulness of preventative interventions. Thromb Res 2010; 125: 166-170.

121. Schaefer WM, Meyer A, Knollmann D. Lung ventilation/perfusion SPECT for diagnosing pulmonary embolism. Is the required minimal $\mathrm{P} / \mathrm{V}$ count rate ratio fulfilled in routine situation? Results from a multicentre survey. Nuklearmedizin 2015; 54: 217-222.

122. Schissler AJ, Rozenshtein A, Schluger NW et al National trends in emergency room diagnosis of pulmonary embolism, 2001-2010: a cross-sectional study. Respir Res 2015; 16: 44.

123. Schuemichen C. Pulmonary embolism: is multislice CT the method of choice? Against. Eur J Nucl Med Mol Imaging 2005; 32: 107-112.

124. Schuemichen C. Perfusion SPECT in patients with suspected pulmonary embolism: how much sensitivity is needed to keep patients alive? Eur J Nucl Med Mol Imaging 2013; 40: 1428-1431.

125. Segal JB, Eng J, Tamariz LJ et al. Review of the evidence on diagnosis of deep venous thrombosis and pulmonary embolism. Ann Fam Med 2007; 5: 63-73.

126. Sen HS, Taylan M, Abakay O et al. Adult diagnosis of Swyer-James-Macleod syndrome: retrospective analysis of four cases. Respir Care 2014; 59: e51-54.

127. Senturk A, Ozsu S, Duru S et al. Prognostic importance of central thrombus in hemodynamically stable patients with pulmonary embolism. Cardiol J 2017; 24: 508-514.

128. Sheh SH, Bellin E, Freeman KD et al. Pulmonary embolism diagnosis and mortality with pulmonary $\mathrm{CT}$ angiography versus ventilation-perfusion scintigraphy: evidence of overdiagnosis with CT? AJR Am J Roentgenol 2012; 198: 1340-1345

129. Shiraev TP, Omari A, Rushworth RL. Trends in pulmonary embolism morbidity and mortality in Australia. Thromb Res 2013; 132: 19-25.

130. Skarlovnik A, Hrastnik D, Fettich J et al. Lung scintigraphy in the diagnosis of pulmonary embolism: current methods and interpretation criteria in clinical practice. Radiol Oncol 2014; 48: 113-119.

131. Smith-Bindman R, Wang Y, Yellen-Nelson TR et al. Predictors of CT Radiation Dose and Their Effect on Patient Care: A Comprehensive Analysis Using Automated Data. Radiology 2016: 151391.

132. Sogaard KK, Schmidt M, Pedersen L et al. 30-year mortality after venous thromboembolism: a population-based cohort study. Circulation 2014; 130: 829-836. 
133. Soler X, Hoh CK, Test VJ et al. Single photon emission computed tomography in chronic thromboembolic pulmonary hypertension. Respirology 2011; 16: 131-137.

134. Soler X, Kerr KM, Marsh JJ et al. Pilot study comparing SPECT perfusion scintigraphy with CT pulmonary angiography in chronic thromboembolic pulmonary hypertension. Respirology 2012; 17: 180-184.

135. Suga K, Kawakami Y, Zaki M et al. Clinical utility of co-registered respiratory-gated $(99 \mathrm{~m}) \mathrm{Tc}$ Technegas/MAA SPECT-CT images in the assessment of regional lung functional impairment in patients with lung cancer. Eur J Nucl Med Mol Imaging 2004; 31: 1280-1290.

136. Sweet PH, 3rd, Armstrong T, Chen J et al. Fatal pulmonary embolism update: 10 years of autopsy experience at an academic medical center. JRSM Short Rep 2013; 4; 2042533313489824

137. Theilade J, Winkel BG, Holst AG et al. A nationwide, retrospective analysis of symptoms, comorbidities, medical care and autopsy findings in cases of fatal pulmonary embolism in younger patients. J Thromb Haemost 2010; 8: 1723-1729.

138. Toney LK, Wanner M, Miyaoka RS et al. Improved prediction of lobar perfusion contribution using technetium-99m-labeled macroaggregate of albumin single photon emission computed tomography/computed tomography with attenuation correction. J Thorac Cardiovasc Surg 2014; 148: 2345-2352.

139. Tunariu N, Gibbs SJ, Win Z et al. Ventilationperfusion scintigraphy is more sensitive than multidetector CTPA in detecting chronic thromboembolic pulmonary disease as a treatable cause of pulmonary hypertension. J Nucl Med 2007; 48: 680-684.
140. van der Hulle T, den Exter PL, Planquette B et al. Risk of recurrent venous thromboembolism and major hemorrhage in cancer-associated incidental pulmonary embolism among treated and untreated patients: a pooled analysis of $926 \mathrm{pa}$ tients. J Thromb Haemost 2016; 14: 105-113.

141. van Es J, den Exter PL, Kaptein AA et al. Quality of life after pulmonary embolism as assessed with SF-36 and PEmb-QoL. Thromb Res 2013, 132: 500-505.

142. van Es J, Douma RA, Hezemans RE et al. Accuracy of X-ray with perfusion scan in young patients with suspected pulmonary embolism. Thromb Res 2015; 136: 221-224.

143. van Mens TE, Scheres LJ, de Jong PG et al. Imaging for the exclusion of pulmonary embolism in pregnancy. Cochrane Database Syst Rev 2017; 1: CD011053.

144. van Ommen $\mathrm{CH}$, Peters M. Acute pulmonary embolism in childhood. Thromb Res 2006; 118 : 13-25.

145. Vedovati MC, Becattini C, Agnelli G et al. Multidetector CT scan for acute pulmonary embolism: embolic burden and clinical outcome. Chest 2012; 142: 1417-1424.

146. Weeda ER, Peacock WF, Fermann GJ et al. Outcomes associated with observation stays versus inpatient admissions for pulmonary embolism. J Thromb Thrombolysis 2016; 42: 513-519.

147. Wells PS, Anderson DR, Rodger M et al. Excluding pulmonary embolism at the bedside without diagnostic imaging: management of patients with suspected pulmonary embolism presenting to the emergency department by using a simple clinical model and d-dimer. Ann Intern Med 2001; 135: 98-107.

148. Wiener RS, Schwartz LM, Woloshin S. Time trends in pulmonary embolism in the United
States: evidence of overdiagnosis. Arch Intern Med 2011; 171: 831-837.

149. Wilkens H, Konstantinides S, Lang I et al. [Chronic thromboembolic pulmonary hypertension: Recommendations of the Cologne Consensus Conference 2016]. Dtsch Med Wochenschr 2016; 141: S62-S69.

150. Wilkens $\mathrm{H}$, Lang $\mathrm{I}$, Blankenburg $\mathrm{T}$ et al. [Chronic thromboembolic pulmonary hypertension--a position paper]. Dtsch Med Wochenschr 2014; 139: 2204-2206.

151. Woussen S, Lopez-Rendon X, Vanbeckevoort D et al. Clinical indications and radiation doses to the conceptus associated with CT imaging in pregnancy: a retrospective study. Eur Radiol 2016; 26: 979-985.

152. Yoo HH, Queluz TH, El Dib R. Anticoagulant treatment for subsegmental pulmonary embolism. Cochrane Database Syst Rev 2016: CD010222.

153. Zanca F, Demeter M, Oyen R et al. Excess radiation and organ dose in chest and abdominal CT due to CT acquisition beyond expected anatomical boundaries. Eur Radiol 2012; 22: 779-788.

154. Zeeb H, Blettner M, Langner I et al. Mortality from cancer and other causes among airline cabin attendants in Europe: a collaborative cohort study in eight countries. Am J Epidemiol 2003; 158: 35-46.

155. Zhou C, Chan HP, Sahiner B et al. Computeraided detection of pulmonary embolism in computed tomographic pulmonary angiography (CTPA): performance evaluation with independent data sets. Med Phys 2009; 36: 3385-3396.

156. Zwierzina D, Limacher A, Mean $\mathrm{M}$ et al. Prospective comparison of clinical prognostic scores in elder patients with a pulmonary embolism. Thromb Haemost 2012; 10: 2270-2276. 


\section{Zertifizierte Fortbildung}

\section{Fragen zum Thema „Lungenszintigraphie”}

1. Welche Antwort trifft nicht zu? Bei der Diagnostik der akuten Lungenembolie (LE) ist die Ventilations/Perfusions (V/P)-SPECT der CTPA vorzuziehen:

a) Bei eingeschränkter Nierenfunktion

b) Bei Hyperthyreose

c) Bei Kontrastmittelrisiken

d) In der Schwangerschaft bei jungen Frauen

e) Bei intensivpflichtigen Patienten

2. Die Ventilationsszintigraphie wird nach Inhalation von Radioaerosol durchgeführt. Eine zentrale Deposition bei Atemwegsobstruktion kann minimiert werden durch:

a) Mittelfeines Aerosol (flüssige oder solide Partikel)

b) Mittelfeines Aerosol (flüssige oder solide Partikel) in Kombination mit einem schnellen Inhalationsmanöver

c) Ein schnelles Inhalationsmanöver

d) Technegas in Kombination mit einem langsamen Inhalationsmanöver

e) Technegas in Kombination mit einem schnellen Inhalationsmanöver

3. Kontamination des Untersuchungsraums durch Radioaerosol kann minimiert werden durch:

a) Anlegen eines Mundschutzes für Personal und Patient

b) Inhalation in einem separaten Raum mit Abzug

c) Ein Hustenmanöver

d) Patient weiter durch den Technegasgenerator ausatmen lassen

e) Durch keine Maßnahme möglich

4. Die Perfusionsszintigraphie erfolgt nach Mikroembolisation der Arteriolen in der Lunge, dabei sollen weniger als $0,1 \%$ der Gefäße embolisiert werden.

a) Das Verfahren ist unter allen Umständen sicher

b) Kindern und Erwachsene erhalten dieselbe Partikelzahl

c) Kindern und Erwachsene erhalten dieselbe Aktivitätsmenge für die SPECT

d) Bei Jugendlichen und Kindern soll die Partikelzahl dem Gewicht angepasst und bei pulmonaler Hypertonie und Rechts/Linksshunt die Partikelzahl reduziert werden e) Bei pulmonaler Hypertonie ist die Perfusionsszintigraphie kontraindiziert

5. Um eine ausreichende Trennung zwischen Ventilation und Perfusion zu gewährleisten, ist ein Aktivitätsverhältnis Ventilation/Perfusion von:

a) Im Mittel 2:1 im klinischen Alltag ausreichend

b) Im Mittel 1:1 im klinischen Alltag ausreichend

c) Im Mittel 1:2 im klinischen Alltag ausreichend

d) Im Mittel 1:3 im klinischen Alltag ausreichend

e) Im Minimum 1:4 anzustreben und ein Verhältnis von 1:3 im klinischen Alltag als noch tolerable Untergrenze anzusehen

6. Bei der Radioaerosolinhalation wurde versehentlich zu viel Aktivität appliziert. Um ein ausreichendes Aktivitätsverhältnis von Ventilation/Perfusion zu gewährleisten, müsste die Tc-99m-MAA-Aktivität über den diagnostischen Höchstwert hinaus angehoben werden.

a) Die Referenzaktivität für Tc-99m-MAA darf aus Gründen der Qualitätssicherung überschritten werden

b) Der Höchstwert darf aus Gründen der Strahlenschutzes auf keinen Fall überschritten werden

c) Die Untersuchung muss auf den nächsten Tag verschoben werden

d) Der Patient wird aufgefordert zu hyperventilieren um das überschüssige Aerosol abzuatmen

e) Die Untersuchung darf nicht mehr fortgesetzt werden

7. Eine Lungenembolie wird anhand der Ventilations/Perfusions (V/P)-SPECT der Lungen diagnostiziert,

a) Sobald ein V/P-mismatch vorliegt, der den anatomischen Grenzen folgt und der von der Größe her den Mindestanforderungen genügt

b) Wenn eine regionale Perfusionsstörung vorliegt

c) Wenn eine unauffällige Ventilation besteht

d) Sobald ein V/P-match vorliegt, der in seiner Form den anatomischen Grenzen folgt (Lappen, Segment, Subsegment)
CME-Fortbildung online

Die Teilnahme an dieser durch die Ärztekammer Nordrhein zertifizierten CME-Fortbildung ist für 12 Monate ausschließlich online möglich. Zur Anmeldung gehen Sie bitte auf https://cme.thieme.de. Dort erfahren Sie auch den genauen Teilnahmeschluss. Es ist immer nur eine Antwort pro Frage zutreffend. Als Abonnent der Nuklearmedizin nehmen Sie kostenlos an deren CME-Programm teil. Je nach CMEFortbildung erhalten Sie bis zu vier Fortbildungspunkte. Weitere Informationen zur Anmeldung und Registrierung finden Sie unter https://cme.thieme.de. Sie erhalten bei inhaltlichen und technischen Fragen tutoriellen Support.

VNR 2760512018154652903

e) Wenn die Ventilation segmental gestört und die Perfusion dort erhalten ist

8. Mit V/P-SPECT der Lungen lassen sich folgende Nebendiagnosen stellen?

a) Es lassen sich keine Nebendiagnosen stellen

b) Nur obstruktive Atemwegerkrankung

c) Nur obstruktive Atemwegerkrankung und Pneumonie

d) Obstruktive Atemwegerkrankung , Pneumonie und Linksherzinsuffizienz

e) Links/Rechts Shunt

9. Bei einem Rechts/Links-Shunt zeigt sich im Ganzkörperszintigramm eine erhöhte Aktivität

a) Nur in den Nieren

b) Nur im Hirn

c) In den Nieren und im Hirn

d) Nur in der Blase

e) Nur in den Speicheldrüsen

10. Welche Antwort trifft nicht zu? Fragestellungen für die Lungenszintigraphie sind

a) Ausschluss einer akuten Lungenembolie (LE).

b) Nachweis eines atypischen Lungenkarzinoids

c) Diagnostik und Differenzierung angeborener Anomalien der Lunge

d) Seitengetrennte Quantifizierung von Lungenventilation und -perfusion präoperativ

e) Nachweis von obstruktiven Belüftungsstörungen (COPD) 